\title{
Generating string solutions in BTZ
}

\author{
Justin R. David, ${ }^{a}$ Chrysostomos Kalousios $^{b, c}$ and Abhishake Sadhukhan ${ }^{a}$ \\ ${ }^{a}$ Centre for High Energy Physics, Indian Institute of Science, \\ C.V. Raman Avenue, Bangalore 560012, India \\ ${ }^{b}$ Institut für Physik der Humboldt-Universität zu Berlin, \\ Newtonstraße 15, D-12489 Berlin, Germany \\ ${ }^{c}$ ICTP South American Institute for Fundamental Research, \\ Instituto de Física Teórica, UNESP-Universidade Estadual Paulista, \\ R. Dr. Bento T. Ferraz 271 - Bl. II, 01140-070, São Paulo, SP, Brasil \\ E-mail: justin@cts.iisc.ernet.in, ckalousi@ift.unesp.br, \\ abhishake@cts.iisc.ernet.in
}

ABSTRACT: Integrability of classical strings in the BTZ black hole enables the construction and study of classical string propagation in this background. We first apply the dressing method to obtain classical string solutions in the BTZ black hole. We dress time like geodesics in the BTZ black hole and obtain open string solutions which are pinned on the boundary at a single point and whose end points move on time like geodesics. These strings upon regularising their charge and spins have a dispersion relation similar to that of giant magnons. We then dress space like geodesics which start and end on the boundary of the BTZ black hole and obtain minimal surfaces which can penetrate the horizon of the black hole while being pinned at the boundary. Finally we embed the giant gluon solutions in the BTZ background in two different ways. They can be embedded as a spiral which contracts and expands touching the horizon or a spike which originates from the boundary and touches the horizon.

KEywords: Integrable Equations in Physics, AdS-CFT Correspondence, Black Holes

ARXIV EPRINT: 1211.5382 


\section{Contents}

1 Introduction 1

2 The BTZ dressing method 3

3 Dressing time like geodesics $\quad 8$

$\begin{array}{ll}3.1 \text { The dressed solution } & 10\end{array}$

4 Dressing space like geodesics $\quad 14$

$\begin{array}{lll}4.1 \text { Closed string solutions } & 20\end{array}$

$5 \quad$ Giant gluons in BTZ $\quad 23$

$\begin{array}{llr}6 & \text { Conclusions } & 29\end{array}$

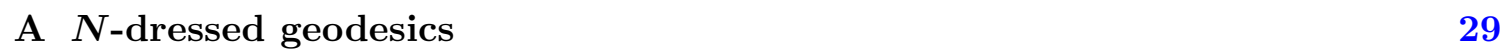

\section{Introduction}

Classical solutions of strings moving in $A d S_{5} \times S^{5}$ backgrounds have played an important role in the AdS/CFT correspondence. Spinning strings in AdS or the sphere have been established as excitations dual to operators with large spins or R-charges respectively [1]. These solutions were crucial in discovering the role of integrability and verifying many of its predictions in the AdS/CFT correspondence, see [2] for a review and a comprehensive list of references. Conversely integrability of strings in AdS was used to generate many new and useful solutions dual to single trace operators as well as Wilson loops in the field theory dual [3-6].

The $A d S_{3} \times S^{3}$ dual pair is another example where the role of integrability is beginning to be investigated [7-14]. One of the most interesting aspects of this dual pair is that classical string propagation in the background of $A d S_{3}$ black holes is integrable unlike the higher dimensional examples [15]. Integrability can be used to classify and generate new classical string solutions in the BTZ background. This in turn will shed light on aspects of black hole physics which can be probed by extended objects. In this paper we apply the dressing method introduced in the AdS/CFT context by [3], to generate and study new solutions of classical strings in the BTZ background.

Trajectories of point like objects described by geodesics are the canonical probes of the causal structure of the black hole. Space like geodesics in black hole backgrounds are used to obtain semi-classical limits of two point correlators in the boundary [16-20] and to study entanglement entropy of the boundary theory for 3 dimensional backgrounds [21]. Studying the behaviour of extended objects gives access to new phenomena near black 
hole horizons. For instance general arguments indicate that strings are expected to spread and become tensionless near black hole horizons due to quantum fluctuations [22-24]. Furthermore minimal surfaces whose boundary are pinned at asymptotic infinity are dual to Wilson/Polyakov loops [25, 26] and are useful probes of the transition from thermal AdS to a black hole in AdS [26, 27] They are also used to evaluate entanglement entropy [21]. See [28] for a nice review and also for some interesting properties of these minimal surfaces. Finally studying spinning strings in the background of black holes in AdS provides clues of the spectrum of the excitations in the dual thermal CFT analogous to the information provided by the spectrum of quasi-normal modes of fields in the black hole background.

The simplest kind of classical string solutions are those which are circular and which wind around the horizon and which then eventually fall into the horizon. Such solutions in the context of the BTZ black holes were studied in [9]. They were classified in terms of the finite gap solutions of the BTZ sigma model. However since the BTZ sigma model is integrable it is possible to apply the dressing method to construct more general classical solutions given a seed solution. In this paper we show how the dressing method developed for the $\mathrm{SU}(1,1)$ principal chiral model $[5,6]$ can be used to generate classical string solutions for the sigma model on $\mathrm{BTZ} \times S^{1}$. One of the by-products of this study is the proof that the dressing method preserves the Virasoro constraints of the seed solution.$^{1}$ This method of generating solutions for the sigma model is different from that obtained by the spectral flow method used to obtain long strings in the SL $(2, R)$ WZW model in [29]. The Wess-Zumino term which allowed for the possibility of obtaining new solutions using the spectral flow in the $\mathrm{SL}(2, R)$ WZW model is not present in the sigma model on $\mathrm{BTZ} \times S^{1}$ considered in this paper.

We first apply the dressing method on time like geodesics to obtain classical string configurations. We obtain open string configurations which are pinned at the boundary but cross the horizon. The end points of these strings move on time like geodesics which have the same constants of motion as the seed geodesic but different initial condition. After a suitable regularization of the energy $E$ and spin $S$ of these solutions which involves subtracting the energy and spin density of seed geodesic we find that their dispersion relation is by the form

$$
E-S=\kappa|\sin \theta|
$$

where $\kappa$ is a function of the background and $\theta$ is the phase of the dressing parameter. Thus the dispersion relation resembles that of the giant magnons found in [30]. We next examine the minimal surfaces obtained by dressing space like geodesics. We show that it is possible to obtain closed strings, they also have a dispersion relation given in (1.1). These surfaces are pinned at two points on the boundary. These points move on time like trajectories at the boundary.

We finally examine the embedding of the well studied giant gluon solutions of [31, 32] in the BTZ background. These are Euclidean worldsheet solutions. We examine two possible embeddings: in one case these solutions have vanishing energy and spin. The configuration

\footnotetext{
${ }^{1}$ The authors are not aware of a proof that the dressing method in general preserves the Virasoro constraints in the existing literature.
} 
is a spiral which originates from the boundary, contracts and touches the horizon and then expands back to the boundary. In the second solution the embedding solutions has a dispersion relation given by

$$
E+S=\kappa \log S .
$$

These are spinning spikes which originate from the boundary and touch the horizon.

The organization of the paper is as follows. In the next section we adapt the dressing method developed for the SU(1,1) sigma model to the BTZ background and show that the Virasoro constraints are preserved by the dressing. In section 3 we dress time like geodesics and discuss the properties of the solutions obtained. In section 4 we repeat this analysis for the case of space like geodesics. In section 5 we embed the giant gluon solutions in the BTZ background and examine its properties. Section 6 contains our conclusions. Appendix A contains the discussion of the general method to obtain multi-dressed solutions in the BTZ background.

\section{The BTZ dressing method}

To apply the dressing method to classical solutions in the BTZ background, we first review the construction of the BTZ black hole as an orbifold of the $A d S_{3}$ hyperboloid. Consider the hyperboloid given by

$$
-u^{2}-v^{2}+x^{2}+y^{2}=-1 .
$$

We then parameterize the hyperboloid as

$$
\begin{array}{ll}
u+x=\cosh \gamma e^{\tilde{\phi}}, & u-x=\cosh \gamma e^{-\tilde{\phi}}, \\
y+v=\sinh \gamma e^{\tilde{t}}, & y-v=\sinh \gamma e^{-\tilde{t}} .
\end{array}
$$

The induced metric on the hyperboloid is given by

$$
d s^{2}=d \gamma^{2}+\cosh ^{2} \gamma d \tilde{\phi}^{2}-\sinh ^{2} \gamma d \tilde{t}^{2} .
$$

The BTZ black hole is then obtained by the identification $[33,34]$

$$
\tilde{t} \sim \tilde{t}-2 \pi r_{-}, \quad \tilde{\phi} \sim \tilde{\phi}+2 \pi r_{+},
$$

where $r_{+}, r_{-}$are the inner and outer radii of the BTZ black hole. The relationship between these coordinates and the conventional radial, time and angular coordinates are given by

$$
\tanh ^{2} \gamma=\frac{r^{2}-r_{+}^{2}}{r^{2}-r_{-}^{2}}, \quad \tilde{t}=r_{+} t-r_{-} \phi, \quad \tilde{\phi}=-r_{-} t+r_{+} \phi
$$

The identifications given in (2.4) ensure that the angular variable $\phi$ has the required periodicity of $2 \pi$. Note that this parametrization is suitable for $r>r_{+}$. Suitable parameterizations exist for all the regions of the black hole. In this paper we will be focussing on the region outside the horizon. For the BTZ background, it is natural to think of the hyperboloid given in $(2.1)$ as an $\mathrm{SL}(2, R)$ group manifold. The group element is given by

$$
g=\left(\begin{array}{ll}
u+x & y+v \\
y-v & u-x
\end{array}\right) .
$$


Then the identification given in (2.4) can be written as

$$
g \sim \tilde{A} g A, \quad \tilde{A}=\left(\begin{array}{cc}
e^{\left(r_{+}-r_{-}\right) \pi} & 0 \\
0 & e^{-\left(r_{+}-r_{-}\right) \pi}
\end{array}\right), \quad A=\left(\begin{array}{cc}
e^{\left(r_{+}-+r_{-}\right) \pi} & 0 \\
0 & e^{-\left(r_{+}+r_{-}\right) \pi}
\end{array}\right) .
$$

These embedding coordinates are related to the conventional coordinates $r, t$ and $\phi$ by the following equations

$$
\begin{aligned}
& r=\sqrt{\left(r_{+}^{2}-r_{-}^{2}\right)\left(y^{2}-v^{2}\right)+r_{+}^{2}}, \quad \phi=\frac{r_{+} \log \left(\frac{u+x}{u-x}\right)+r_{-} \log \left(\frac{y+v}{y-v}\right)}{2\left(r_{+}^{2}-r_{-}^{2}\right)}, \\
& t=\frac{r_{-} \log \left(\frac{u+x}{u-x}\right)+r_{+} \log \left(\frac{y+v}{y-v}\right)}{2\left(r_{+}^{2}-r_{-}^{2}\right)} .
\end{aligned}
$$

Thus the action of the string propagating in BTZ times $S^{1}$ is given by

$$
S=-\frac{\hat{\lambda}}{2} \int d^{2} \sigma\left(\frac{1}{2} \operatorname{Tr}\left(g^{-1} \partial_{a} g g^{-1} \partial^{a} g^{-1}\right)+\partial_{a} Z \partial^{a} Z\right),
$$

together with the identifications given in (2.7). Here $Z$ is the coordinate along the $S^{1}$ and $\lambda$ is the coupling of the sigma model. Translational symmetry along the time direction $t$ and the angular direction $\phi$ give rise to the global charges $E$ and $S$. These charges have the following simple relation in terms of the right and left currents of the sigma model

$$
\begin{aligned}
& E+S=\frac{\hat{\lambda}}{2}\left(r_{+}-r_{-}\right) \int_{0}^{2 \pi} d \sigma \operatorname{Tr}\left(\partial_{0} g g^{-1} \sigma^{3}\right), \\
& E-S=-\frac{\hat{\lambda}}{2}\left(r_{+}+r_{-}\right) \int_{0}^{2 \pi} d \sigma \operatorname{Tr}\left(g^{-1} \partial_{0} g \sigma^{3}\right) .
\end{aligned}
$$

The equations of motion of the sigma model are given by

$$
\partial^{a}\left(\partial_{a} g g^{-1}\right)=0, \quad \partial^{a} \partial_{a} Z=0 .
$$

where $a \in\{0,1\}$ refers to the worldsheet coordinates $\tau, \sigma$. It is convenient to define the light-cone coordinates as

$$
\sigma_{ \pm}=\frac{1}{2}(\tau \pm \sigma), \quad \partial_{ \pm}=\partial_{\tau} \pm \partial_{\sigma}
$$

Let us choose a gauge in which

$$
Z=\frac{\hat{J}}{2 \pi \lambda} \tau+\hat{m} \sigma
$$

where $\hat{J}$ refers to the momentum on $S^{1}$ and $\hat{m}$ refers to the winding. We will restrict our attention to classical solutions with $\hat{m}=0$. Then the Virasoro constraints of the sigma model reduce to

$$
\frac{1}{2} \operatorname{Tr}\left(j_{ \pm}^{2}\right)=-\left(\frac{\hat{J}}{2 \pi \hat{\lambda}}\right)^{2}
$$


where

$$
\hat{j}_{ \pm}=\partial_{ \pm} g g^{-1} .
$$

Our goal now is to find a method to generate new solutions for this sigma model from a given solution. For this we adopt the following strategy. Consider a solution of the $\mathrm{SL}(2, R)$ sigma model which is parameterized as given in (2.6). We use the isomorphism between $\mathrm{SL}(2, R)$ and $\mathrm{SU}(1,1)$ and consider it as solution in a $\mathrm{SU}(1,1)$ sigma model by the parametrization

$$
\hat{g}=\left(\begin{array}{cc}
u-i v & x+i y \\
x-i y & u+i v
\end{array}\right)=Q g Q^{-1},
$$

where

$$
Q=\frac{1}{\sqrt{2}}\left(\begin{array}{cc}
1 & i \\
1 & -i
\end{array}\right) .
$$

From the fact that $g \in \mathrm{SL}(2, R)$ it is easy to show that $\hat{g}$ satisfies the defining property of $\mathrm{SU}(1,1)$ which is given by

$$
\hat{g}^{\dagger} M \hat{g}=M, \quad \operatorname{det} \hat{g}=1, \quad M=\left(\begin{array}{cc}
1 & 0 \\
0 & -1
\end{array}\right) .
$$

It is clear from the relationship between the $\mathrm{SU}(1,1)$ and the $\mathrm{SL}(2, R)$ group elements given in (2.16) that a solution to the classical equations of motion and the Virasoro constraints of the $\mathrm{BTZ} \times S^{1}$ sigma model will be a solution to the classical equations of motion and the Virasoro constraints of the $\mathrm{SU}(1,1) \times S^{1}$ sigma model.

Now that we have a solution in the $\mathrm{SU}(1,1) \times S^{1}$ sigma model we can use the dressing method developed for this sigma model in $[5,6]$ to generate new classical solutions to this sigma model. We then transform it back to a solution in the $\mathrm{BTZ} \times S^{1}$ sigma model. This will then be a new solution to the BTZ $\times S^{1}$ sigma model. In general the solutions generated by this method will be classical open strings moving in the BTZ background.

Before we proceed to explicitly apply this strategy we review the dressing method for the $\mathrm{SU}(1,1) \times S^{1}$ sigma model. Consider the equations of motion to this sigma model which are given by

$$
\partial_{+}\left(\partial_{-} \hat{g} \hat{g}^{-1}\right)+\partial_{-}\left(\partial_{+} \hat{g} \hat{g}^{-1}\right)=0 .
$$

To generate new solutions from a given solution we consider the following system of equations

$$
i \partial_{+} \Psi=\frac{A \Psi}{1-\lambda}, \quad i \partial_{-} \Psi=\frac{B \Psi}{1+\lambda},
$$

where $\lambda$ is the complex spectral parameter and $A, B$ are independent of $\lambda$. Let us suppose we have a solution to the equations of motion given in (2.19). Then taking

$$
A=i \partial_{+} \hat{g} \hat{g}^{-1}, \quad B=i \partial_{-} \hat{g} \hat{g}^{-1},
$$

guarantees that the integrability constraints of the system of equations in (2.20) is satisfied. This is because the equation in (2.19) together with the identity

$$
\partial_{-}\left(\partial_{+} \hat{g} \hat{g}^{-1}\right)-\partial_{+}\left(\partial_{-} \hat{g} \hat{g}^{-1}\right)-\left[\partial_{1} \hat{g} \hat{g}^{-}, \partial_{+} \hat{g} \hat{g}^{-1}\right]=0,
$$


are the integrability constraints of the system of equations in (2.20). Thus for this situation the system $(2.20)$ can be solved to obtain $\Psi(\lambda)$ with

$$
\Psi(0)=\hat{g}
$$

Now given the consistent system of equations in (2.20) which implies that the integrability constraints are satisfied, it is easy to see that $\Psi(0)$ is assured to satisfy the equation of motion (2.19). This is because the equation of motion is part of the integrability constraint of the system in (2.20). Now to ensure that we obtain a solution in the group $\mathrm{SU}(1,1)$ we impose the following constraint

$$
\Psi^{\dagger}(\bar{\lambda}) M \Psi(\lambda)=M
$$

Given that $A, B$ are constructed as in (2.21) from a known solution to (2.20), we can generate a new solution by considering a $\lambda$-dependent gauge parameter $\chi(\lambda)$. Under this transformation we obtain the system of equations as in (2.20) but with $\Psi^{\prime}, A^{\prime}, B^{\prime}$ given by

$$
\begin{aligned}
& \Psi^{\prime}=\chi \Psi, \\
& A^{\prime}=\chi A \chi^{-1}+i(1-\lambda) \partial_{+} \chi \chi^{-1}, \\
& B^{\prime}=\chi B \chi^{-1}+i(1+\lambda) \partial_{-} \chi \chi^{-1} .
\end{aligned}
$$

If we ensure that $A^{\prime}, B^{\prime}$ are independent of $\lambda$, then it is guaranteed that $\hat{g}^{\prime}=\Psi^{\prime}(0)$ is a possible new solution to the set of equations in $(2.19)$. The $\mathrm{SU}(1,1)$ constraint in $(2.24)$ requires $\chi$ to satisfy the equation

$$
\chi^{\dagger}(\bar{\lambda}) M \chi(\lambda)=M
$$

To fix the form of $\chi$ so that $A^{\prime}, B^{\prime}$ are independent of $\lambda$ we proceed as follows. The form of $\chi$ is taken to be as

$$
\chi(\lambda)=1+\frac{\lambda_{1}-\bar{\lambda}_{1}}{\lambda-\lambda_{1}} P
$$

where $P$ is a projection operator given by

$$
P=\frac{\Psi\left(\bar{\lambda}_{1}\right) e e^{\dagger} \Psi^{\dagger}\left(\bar{\lambda}_{1}\right) M}{e^{\dagger} \Psi^{\dagger}\left(\bar{\lambda}_{1}\right) M \Psi\left(\bar{\lambda}_{1}\right) e}
$$

where $e$ is a constant vector and $\lambda_{1}$ is an arbitrary complex parameter. This projection operators satisfies the conditions

$$
P^{2}=P, \quad M P^{\dagger} M=P .
$$

Note that the determinant of $\chi(0)$ is given by

$$
\operatorname{det} \chi(0)=\frac{\bar{\lambda}_{1}}{\lambda_{1}}
$$

Thus to ensure that the new solutions which we call the dressed solution belongs to $\mathrm{SU}(1,1)$, $\hat{g}^{\prime}$ is given by

$$
\hat{g}^{\prime}=\sqrt{\frac{\lambda_{1}}{\bar{\lambda}_{1}}} \chi(0) \Psi(0)=\sqrt{\frac{\lambda_{1}}{\bar{\lambda}_{1}}} \chi(0) \hat{g}(0) .
$$


We will now show that the form of $\chi$ given in (2.27) ensures that $A^{\prime}, B^{\prime}$ are independent of $\lambda$. From our analysis in the previous paragraphs we see that this is required so that the equations of motion of the sigma model is satisfied by $\Psi^{\prime}(0)$. Let us first show that $A^{\prime}$ given in (2.25) is independent of $\lambda$. For this we first evaluate

$$
\chi^{-1}(\lambda)=1+\frac{\bar{\lambda}_{1}-\lambda_{1}}{\lambda-\bar{\lambda}_{1}} P
$$

From the expression of $A^{\prime}$ given in (2.25) and that of $\chi$ and $\chi^{-1}$ in (2.27) and (2.32) we see that the elements of $A^{\prime}$ are holomorphic functions of $\lambda$ except for the possible poles at $\lambda=\lambda_{1}$ and $\lambda=\bar{\lambda}_{1}$. We will now show that the residue at the possible pole at $\lambda=\lambda_{1}$ vanishes. Taking the limit $\lambda \rightarrow \lambda_{1}$ we obtain

$$
A^{\prime}\left(\lambda \rightarrow \lambda_{1}\right)=\frac{\lambda_{1}-\bar{\lambda}_{1}}{\lambda-\lambda_{1}}\left[P A(1-P)+i\left(1-\lambda_{1}\right) \partial_{+} P(1-P)\right] .
$$

Now using the equations of motion in (2.20) and the definition of $P$ in (2.28), we see that

$$
i \partial_{+} P=\frac{A P}{\left(1-\bar{\lambda}_{1}\right)}-\frac{P A}{1-\lambda_{1}}-\frac{\bar{\lambda}_{1}-\lambda_{1}}{\left(1-\lambda_{1}\right)\left(1-\bar{\lambda}_{1}\right)} P \operatorname{Tr}(A P)
$$

Here we have used the equation

$$
A^{\dagger} M=M A,
$$

which can be obtained by differentiating the $\mathrm{SU}(1,1)$ constraint in $(2.18)$. To obtain the last term in (2.34) we have used the identity

$$
\operatorname{Tr}(A P)=\frac{e^{\dagger} \Psi(\bar{\lambda})^{\dagger} M A \psi(\bar{\lambda}) e}{e^{\dagger} \Psi^{\dagger}\left(\bar{\lambda}_{1}\right) M \Psi\left(\bar{\lambda}_{1}\right) e}
$$

which can be shown easily by taking the trace in the following orthonormal basis

$$
v_{1}=\frac{M \Psi\left(\bar{\lambda}_{1}\right) e}{\sqrt{e^{\dagger} \Psi^{\dagger}\left(\bar{\lambda}_{1}\right) \Psi\left(\bar{\lambda}_{1}\right) e}}, \quad v_{2}=v_{\perp},
$$

where $v_{\perp}$ is the orthogonal unit vector perpendicular to $v_{1}$. Using this basis one can also show that $\operatorname{Tr} P=1$. Now substituting $i \partial_{+} P$ from (2.34) into the expression for $A^{\prime}$ in the limit $\lambda \rightarrow \lambda_{1}$ given in (2.33), we see the residue at $\lambda_{1}$ vanishes. One can perform the same analysis for the limit $\lambda \rightarrow \bar{\lambda}_{1}$ and show that the potential pole at $\bar{\lambda}_{1}$ in $A^{\prime}$ also vanishes. This implies that $A^{\prime}$ is a meromorphic function in the $\lambda$ plane which approaches the matrix $A$ at $\lambda \rightarrow \infty$. Thus Liouville's theorem in complex analysis allows us to conclude that $A^{\prime}$ is independent of $\lambda$. A similar analysis for the matrix $B^{\prime}$ can be used to show that $B^{\prime}$ is independent of $\lambda$. Therefore the dressed solution given in (2.31) is guaranteed to solve the equations of motion of the $\mathrm{SU}(1,1)$ sigma model.

Dressing preserves Virasoro constraints. We now show that the dressed solution also preserves the Virasoro constraints. The original solution satisfies the Virasoro constraints which are given by

$$
\frac{1}{2} \operatorname{Tr}\left(\partial_{ \pm} \hat{g} \hat{g}^{-1}\right)^{2}=-\left(\frac{\hat{J}}{2 \pi \hat{\lambda}}\right)^{2}
$$


Let us examine the first constraint in the above set of equations, a similar analysis applies to the second Virasoro constraint. From the definition of the dressed solution in (2.31) we see that the dressed solution preserves the Virasoro constraints provided the following equation is obeyed by the dressing matrix $\chi$

$$
\operatorname{Tr}\left(2 i A \chi^{-1}(0) \partial_{+} \chi(0)+\partial_{+} \chi^{-1}(0) \partial_{+} \chi(0)\right)=0 .
$$

Now substituting the definition of $\chi$ in (2.27) and its inverse in (2.32) as well as the equation (2.34) we obtain

$$
\operatorname{Tr}\left(2 i A \chi^{-1}(0) \partial_{ \pm} \chi(0)\right)=\frac{2\left(\lambda_{1}-\bar{\lambda}_{1}\right)^{2}}{\lambda_{1} \bar{\lambda}_{1}\left(1-\bar{\lambda}_{1}\right)\left(1-\lambda_{1}\right)}\left(\operatorname{Tr}\left(A^{2} P\right)-(\operatorname{Tr}(A P))^{2}\right) .
$$

Here we have also used the equation

$$
\operatorname{Tr}(A P A P)=(\operatorname{Tr} A P)^{2},
$$

which holds since the projector in (2.28) is a rank 1 projector. It can also be shown explicitly by evaluating the trace using the complete set of states given in (2.37). Now using the same manipulations one can show that

$$
\operatorname{Tr}\left(\partial_{+} \chi^{-1}(0) \partial_{+} \chi(0)\right)=-\frac{2\left(\lambda_{1}-\bar{\lambda}_{1}\right)^{2}}{\lambda_{1} \bar{\lambda}_{1}\left(1-\bar{\lambda}_{1}\right)\left(1-\lambda_{1}\right)}\left(\operatorname{Tr}\left(A^{2} P\right)-(\operatorname{Tr}(A P))^{2}\right) .
$$

The equations (2.40) and (2.42) imply that the equation (2.39) is true and thus the dressed solution also preserves the Virasoro constraints.

\section{$3 \quad$ Dressing time like geodesics}

In this section we apply the dressing method to time like geodesics in the BTZ background and obtain open string solutions. The components of the $\operatorname{SL}(2, R)$ matrix for geodesics in BTZ can be written as

$$
g_{0}=\left(\begin{array}{cc}
a(\tau) \exp (f(\tau)) & b(\tau) \exp (g(\tau)) \\
b(\tau) \exp (-g(\tau)) & a(\tau) \exp (-f(\tau))
\end{array}\right),
$$

with the constraint $a(\tau)^{2}-b(\tau)^{2}=1$. A convenient parametrization for these variables is given by

$$
a(\tau)=\cosh \gamma(\tau), \quad b(\tau)=\sinh \gamma(\tau) .
$$

The Virasoro constraints for the geodesic reduce to

$$
\dot{\gamma}^{2}+\frac{c_{1}^{2}}{\cosh ^{2} \gamma}-\frac{c_{2}^{2}}{\sinh ^{2} \gamma}+\left(\frac{\hat{J}}{2 \pi \hat{\lambda}}\right)^{2}=0,
$$

where the dot denotes the derivative with respect to the worldsheet time coordinate and $c_{1}, c_{2}$ are the constants of motion given by

$$
\dot{f} \cosh ^{2} \gamma=c_{1}, \quad \dot{g} \sinh ^{2} \gamma=c_{2} .
$$


We choose the initial conditions

$$
a(0)=a_{0}, \quad b(0)=b_{0}, \quad f(0)=0, \quad b(0)=0, \quad \dot{\gamma}(0)=0 .
$$

It is easy to show using the equations of motion that for the geodesic solution given in (3.1), the current

$$
j=\partial_{+} g_{0} g_{0}^{-1}=\partial_{-} g_{0} g_{0}^{-1}
$$

is a constant matrix. Therefore the solution to the equation of motion can also be written as

$$
g=\exp (j \tau)\left(\begin{array}{ll}
a_{0} & b_{0} \\
b_{0} & a_{0}
\end{array}\right)
$$

Note that this solution clearly satisfies the initial conditions given in (3.4). Using this solution consider the set of equations

$$
\partial_{+} \Psi_{s}=\frac{j \Psi_{s}}{1-\lambda}, \quad \partial_{-} \Psi_{s}=\frac{j \Psi_{s}}{1+\lambda} .
$$

Since $j$ is a constant, the equations in (3.7) can easily be integrated. Their solution is given by

$$
\Psi_{s}=\exp \left(j \frac{\tau+\sigma \lambda}{1-\lambda^{2}}\right)\left(\begin{array}{ll}
a_{0} & b_{0} \\
b_{0} & a_{0}
\end{array}\right) .
$$

Note that this solution satisfies the initial condition

$$
\Psi_{s}(0)=g
$$

Explicitly performing the exponentiation in the solution (3.8) we obtain

$$
\Psi_{s}(\lambda)=\left(\begin{array}{cc}
a_{0} \cos \vartheta+\frac{c_{1}}{a_{0} J} \sin \vartheta & b_{0} \cos \vartheta+\frac{c_{2}}{b_{0} J} \sin \vartheta \\
b_{0} \cos \vartheta-\frac{c_{2}}{b_{0} J} \sin \vartheta & a_{0} \cos \vartheta-\frac{c_{1}}{a_{0} J} \sin \vartheta
\end{array}\right)
$$

where

$$
\vartheta=\frac{J(\tau+\sigma \lambda)}{1-\lambda^{2}}, \quad J=\frac{\hat{J}}{2 \pi \hat{\lambda}} .
$$

To obtain (3.10) we have also used the Virasoro constraint

$$
\frac{c_{1}^{2}}{a_{0}^{2}}-\frac{c_{2}^{2}}{b_{0}^{2}}+J^{2}=0
$$

Now given a solution to the set of equations in (3.7) it is easy to find the solution to the corresponding $\mathrm{SU}(1,1)$ monodromy equations given in $(2.20)$. Since the relation between $\mathrm{SU}(1,1)$ and $\mathrm{SL}(2, R)$ is given by $(2.16)$ we see that the corresponding solution to $(2.20)$ is given by

$$
\Psi=Q \Psi_{s} Q^{-1}
$$


Writing this out explicitly we obtain

$$
\Psi(\lambda)=\left(\begin{array}{cc}
a_{0} \cos \vartheta-i \frac{c_{2}}{b_{0} J} \sin \vartheta & \frac{c_{1}}{a_{0} J} \sin \vartheta+i b_{0} \cos \vartheta \\
\frac{c_{1}}{a_{0} J} \sin \vartheta-i b_{0} \cos \vartheta & a_{0} \cos \vartheta+i \frac{c_{2}}{b_{0} J} \sin \vartheta
\end{array}\right) .
$$

Now that we have the explicit form of $\Psi(\lambda)$ corresponding to the time like geodesics we can construct $\chi(\lambda)$ as defined in (2.27) and proceed to obtain the dressed solution in the $\mathrm{SL}(2, R)$ sigma model which is given by

$$
g^{\prime}=Q^{-1} \hat{g}^{\prime} Q=Q^{-1} \sqrt{\frac{\lambda_{1}}{\bar{\lambda}_{1}}} \chi(0) \hat{g}(0) Q,
$$

where we have substituted $\hat{g}^{\prime}$ from $(2.31)$.

\subsection{The dressed solution}

In this section we will present an explicit example of a class of classical string solutions obtained by applying the dressing method on time like geodesics. For this we will first simplify the situation by considering time like geodesics which satisfy the following relation

$$
c_{1}=c_{2}=a_{0} b_{0} J
$$

The Virasoro constraint at the initial point $\tau=0$ given in (3.12) is trivially satisfied with the condition given in (3.16). Evaluating the global charges of these time like geodesics we obtain the following

$$
E+S=0, \quad E-S=-2 \pi \hat{\lambda}\left(r_{+}+r_{-}\right)\left(c_{1}+c_{2}\right)=-4 \pi a_{0} b_{0} J
$$

Using the condition (3.16) in the expression for $\Psi(\lambda)$ given in (3.14) we find that it reduces to

$$
\Psi(\lambda)=\left(\begin{array}{cc}
a_{0} \exp (-i \vartheta) & i b_{0} \exp (-i \vartheta) \\
-i b_{0} \exp (i \vartheta) & a_{0} \exp (i \vartheta)
\end{array}\right) .
$$

We will now choose the following constant vector for constructing the dressing factor

$$
e=\left(\begin{array}{l}
1 \\
0
\end{array}\right)
$$

Using the definition of $P$ given in (2.28) we find its elements are given by

$$
\begin{array}{ll}
P_{11}=\frac{a_{0}^{2} \exp \left(-J_{1}\right)}{a_{0}^{2} \exp \left(-J_{1}\right)-b_{0}^{2} \exp \left(J_{1}\right)}, & P_{12}=\frac{-i a_{0} b_{0} \exp \left(-i J_{1}\right)}{a_{0}^{2} \exp \left(-J_{1}\right)-b_{0}^{2} \exp \left(J_{1}\right)}, \\
P_{21}=\frac{-i a_{0} b_{0} \exp \left(i J_{1}\right)}{a_{0}^{2} \exp \left(-J_{1}\right)-b_{0}^{2} \exp \left(J_{1}\right)}, & P_{22}=\frac{-b_{0}^{2} \exp \left(J_{1}\right)}{a_{0}^{2} \exp \left(-J_{1}\right)-b_{0}^{2} \exp \left(J_{1}\right)},
\end{array}
$$

where

$$
\begin{aligned}
i J_{1}=J\left[\frac{\tau+\sigma \lambda_{1}}{1-\lambda_{1}^{2}}-\frac{\tau+\sigma \bar{\lambda}_{1}}{1-\bar{\lambda}_{1}^{2}}\right] & =i 2 J\left[\frac{\tau r^{2} \sin (2 \theta)+r \sigma \sin (\theta)\left(1+r^{2}\right)}{1+r^{4}-2 r^{2} \cos (2 \theta)}\right] \\
J_{2}=J\left[\frac{\tau+\sigma \lambda_{1}}{1-\lambda_{1}^{2}}+\frac{\tau+\sigma \bar{\lambda}_{1}}{1-\bar{\lambda}_{1}^{2}}\right] & =2 J\left[\frac{\tau\left(1-r^{2} \cos (2 \theta)\right)+\sigma r \cos (\theta)\left(1-r^{2}\right)}{1+r^{4}-2 r^{2} \cos (2 \theta)}\right] .
\end{aligned}
$$


Note that the projection matrix has the following property: given a definite value of $\tau$, the asymptotic values of $P$ for $\sigma \rightarrow \pm \infty$ are given by

$$
P(\tau, \infty)=\left(\begin{array}{ll}
0 & 0 \\
0 & 1
\end{array}\right), \quad P(\tau,-\infty)=\left(\begin{array}{ll}
1 & 0 \\
0 & 0
\end{array}\right) .
$$

By using the projection matrix given in (3.20) to construct the normalized dressing factor dressing factor $\hat{\chi}(0)$ we obtain

$$
\begin{array}{ll}
\hat{\chi}_{11}(0)=\exp (i \theta)-\frac{2 i \sin (\theta) a_{0}^{2} \exp \left(-J_{1}\right)}{a_{0}^{2} \exp \left(-J_{1}\right)-b_{0}^{2} \exp \left(J_{1}\right)}, & \hat{\chi}_{12}(0)=-\frac{2 \sin (\theta) a_{0} b_{0} \exp \left(-i J_{2}\right)}{a_{0}^{2} \exp \left(-J_{1}\right)-b_{0}^{2} \exp \left(J_{1}\right)}, \\
\hat{\chi}_{22}(0)=\exp (i \theta)+\frac{2 i \sin (\theta) a_{0}^{2} \exp \left(-J_{1}\right)}{a_{0}^{2} \exp \left(-J_{1}\right)-b_{0}^{2} \exp \left(J_{1}\right)}, & \hat{\chi}_{21}(0)=-\frac{2 \sin (\theta) a_{0} b_{0} \exp \left(i J_{2}\right)}{a_{0}^{2} \exp \left(-J_{1}\right)-b_{0}^{2} \exp \left(J_{1}\right)},
\end{array}
$$

where

$$
\hat{\chi}=\sqrt{\frac{\lambda_{1}}{\bar{\lambda}_{1}}} \chi(0)
$$

and the complex parameter $\lambda_{1}=r e^{i \theta}$. It can be easily verified that the dressing factor in $(3.23)$ belongs to $\mathrm{SU}(1,1)$. Note that when the parameter $\lambda_{1}$ becomes real, that is $\theta=0$, the dressing factor reduces to identity. Thus there is a smooth limit in which the dressed solution will reduce to the original geodesic. Now using the expression in (3.15), the new solution to the sigma model which satisfies the Virasoro constraints is given by

$$
\begin{aligned}
& u^{\prime}=a_{0} \cos (\theta-J \tau)-\frac{2 \sin (\theta)\left(a_{0}^{3} \exp \left(-J_{1}\right) \sin (J \tau)+a_{0} b_{0}^{2} \sin \left(J \tau-J_{2}\right)\right)}{a_{0}^{2} \exp \left(-J_{1}\right)-b_{0}^{2} \exp \left(J_{1}\right)} \\
& v^{\prime}=-a_{0} \sin (\theta-J \tau)+\frac{2 \sin (\theta)\left(a_{0}^{3} \exp \left(-J_{1}\right) \cos (J \tau)-a_{0} b_{0}^{2} \cos \left(J \tau-J_{2}\right)\right)}{a_{0}^{2} \exp \left(-J_{1}\right)-b_{0}^{2} \exp \left(J_{1}\right)} \\
& x^{\prime}=-b_{0} \sin (\theta-J \tau)+\frac{2 \sin (\theta) a_{0}^{2} b_{0}\left(\exp \left(-J_{1}\right) \cos (J \tau)-\cos \left(J \tau-J_{2}\right)\right)}{a_{0}^{2} \exp \left(-J_{1}\right)-b_{0}^{2} \exp \left(J_{1}\right)} \\
& y^{\prime}=b_{0} \cos (\theta-J \tau)-\frac{2 \sin (\theta) a_{0}^{2} b_{0}\left(\exp \left(-J_{1}\right) \sin (J \tau)+\sin \left(J \tau-J_{2}\right)\right)}{a_{0}^{2} \exp \left(-J_{1}\right)-b_{0}^{2} \exp \left(J_{1}\right)}
\end{aligned}
$$

where $J_{1}$ and $J_{2}$ are given by (3.21). We have used the parametrization of $\operatorname{SL}(2, R)$ given in (2.6) to read out the $u^{\prime}, v^{\prime}, x^{\prime}, y^{\prime}$ values of the dressed solution. Note that as a simple check it can be verified that the constraint $-u^{\prime 2}-v^{\prime 2}+x^{\prime 2}+y^{\prime 2}=-1$ is satisfied. We have also explicitly verified that the solution in (3.25) satisfies both the equation of motion as well as the Virasoro constraints given by

$$
-\partial_{ \pm}\left(u^{\prime}+x^{\prime}\right) \partial_{ \pm}\left(u^{\prime}-x^{\prime}\right)+\partial_{ \pm}\left(y^{\prime}+v^{\prime}\right) \partial_{ \pm}\left(y^{\prime}-v^{\prime}\right)=-J^{2}
$$

Multiple dressed solutions can be obtained by the general procedure discussed in the appendix. 
Description of the solution. We now briefly describe the features of the solution. At any given value of the worldsheet time the end points of the string $\sigma \rightarrow \pm \infty$ move as time like geodesics. Taking the limit $\sigma \rightarrow \infty$ in the solution given in (3.25), we obtain

$$
\begin{array}{rlrl}
u^{\prime} & =a_{0} \cos (\theta-J \tau), & v^{\prime}=-a_{0} \sin (\theta-J \tau), \\
x^{\prime}=-b_{0} \sin (\theta-J \tau), & y^{\prime}=b_{0} \cos (\theta-J \tau) .
\end{array}
$$

In taking this limit we have assumed that $\sin \theta>0$ (a similar analysis can be repeated for $\sin \theta<0)$. Comparing this to the seed solution $\Psi_{x}(0)=g$ given in (3.10) we see that it is the geodesic with same constants of motion but with different initial conditions. Similarly taking the limit $\sigma \rightarrow-\infty$ we obtain

$$
\begin{array}{rlrl}
u^{\prime} & =a_{0} \cos (\theta+J \tau), & v^{\prime}=a_{0} \sin (\theta+J \tau), \\
x^{\prime}=b_{0} \sin (\theta+J \tau), & y^{\prime}=b_{0} \cos (\theta+J \tau) .
\end{array}
$$

Again, this is the same geodesic as in (3.27) with $\theta \rightarrow-\theta$. Thus, the end points of the string move as geodesics with the same constants of motion as the seed geodesic, but with initial conditions depending on $\theta$.

To further characterize these solutions we will evaluate their global charges. We first show that the charge $E+S=0$, thus preserving the condition of the seed geodesic given in (3.17). The charge of the dressed solution is given by

$$
\begin{aligned}
\frac{E+S}{r_{+}-r_{-}} & =\frac{\hat{\lambda}}{2} \int_{-\infty}^{\infty} d \sigma \operatorname{Tr}\left(\partial_{0} g^{\prime} g^{\prime-1} \sigma^{3}\right), \\
& =\frac{\hat{\lambda}}{2} \int_{-\infty}^{\infty} d \sigma \operatorname{Tr}\left(\partial_{0} \hat{g}^{\prime} \hat{g}^{\prime-1} Q \sigma^{3} Q^{-1}\right), \\
& =\frac{\hat{\lambda}}{2} \int_{-\infty}^{\infty} d \sigma \operatorname{Tr}\left(\partial_{0} \hat{g}^{\prime} \hat{g}^{\prime-1} \sigma^{1}\right) .
\end{aligned}
$$

In the second and third line of the equation we have converted the $\mathrm{SL}(2, R)$ variables to $\mathrm{SU}(1,1)$. Now from the fact that the dressing method is a gauge transformation the new currents are related to the old ones by $(2.25)$

$$
\begin{aligned}
& A^{\prime}=\chi A \chi^{-1}+i(1-\lambda) \partial_{+} \chi \chi^{-1}, \\
& B^{\prime}=\chi B \chi^{-1}+i(1+\lambda) \partial_{-} \chi \chi^{-1} .
\end{aligned}
$$

We have also seen that $A^{\prime}, B^{\prime}$ are independent of $\lambda$, therefore we can evaluate the l.h.s. of the above equations at any convenient value of $\lambda$. Let us evaluate the l.h.s. at $\lambda \rightarrow \infty$. This results in the following equations

$$
\begin{aligned}
& \partial_{+} \hat{g}^{\prime} \hat{g}^{-1}=\partial_{+} \hat{g} \hat{g}^{-1}-\left(\lambda_{1}-\bar{\lambda}_{1}\right) \partial_{+} P, \\
& \partial_{-} \hat{g}^{\prime} \hat{g}^{\prime-1}=\partial_{-} \hat{g} \hat{g}^{-1}+\left(\lambda_{1}-\bar{\lambda}_{1}\right) \partial_{-} P .
\end{aligned}
$$

Adding both equations we obtain the following

$$
\partial_{0} \hat{g}^{\prime} \hat{g}^{\prime-1}=\partial_{0} \hat{g} \hat{g}^{-1}-\left(\lambda_{1}-\bar{\lambda}_{1}\right) \partial_{\sigma} P .
$$


This equation is very convenient to evaluate the charge given in (3.29) .

$$
\begin{aligned}
\frac{2(E+S)}{\hat{\lambda}\left(r_{+}-r_{-}\right)} & =\int_{-\infty}^{\infty} d \sigma \operatorname{Tr}\left(\partial_{0} \hat{g} \hat{g}^{-1} \sigma^{1}\right)-\left(\lambda_{1}-\bar{\lambda}_{1}\right) \operatorname{Tr}\left[\left(P(\tau, \infty)-P(\tau,-\infty) \sigma^{1}\right]\right. \\
& =0
\end{aligned}
$$

To obtain the last line we have used the fact that the seed geodesic has the property $c_{1}=c_{2}$ and the values of the asymptotic values of the projection matrix are given in (3.22). Thus the dressing does not change the left charge $E+S$. It is easy to see from the analysis that this holds true for any number of dressings of this geodesics provided the asymptotic property of the projection matrix is that given in (3.22). Now let us study the charge $E-S$. Here we need to explicitly perform the integrals. By a tedious calculation, it can be shown that the following integral is given by

$$
\begin{aligned}
\int \operatorname{Tr}\left(g^{\prime-1} \partial_{\tau} g^{\prime} \sigma^{3}\right) d \sigma & =4 a_{0} b_{0}\left[J \sigma+\frac{-2 a_{0}^{2}+\left(1+2 b_{0}^{2}\right) \exp (2 B) \cos (C)}{\left(-1+b_{0}^{2}(-1+\exp (4 B))\right) r} \sin (\theta)\right], \\
B & =\frac{J r\left[\sigma\left(1+r^{2}\right)+2 \tau r \cos (\theta)\right]}{1+r^{4}-2 r^{2} \cos (2 \theta)} \sin \theta \\
C & =\frac{2 J r\left[\sigma\left(r^{2}-1\right) \cos (\theta)+r \tau\left(r^{2}-\cos (2 \theta)\right)\right]}{1+r^{4}-2 r^{2} \cos (2 \theta)} .
\end{aligned}
$$

Now on taking the limits we obtain the following global charge

$$
\begin{aligned}
E-S & =-\frac{\hat{\lambda}}{2}\left(r_{+}+r_{-}\right) \lim _{L \rightarrow \infty} \int_{-L}^{L} d \sigma \operatorname{Tr}\left(g^{-1} \partial_{\tau} g \sigma^{3}\right), \\
& =-\frac{\hat{\lambda}}{2}\left(r_{+}+r_{-}\right)\left(8 a_{0} b_{0} J L-8 \frac{a_{0} b_{0}|\sin \theta|}{r}\right) .
\end{aligned}
$$

Thus the leading contribution to the global charge diverges linearly with the worldsheet length. From (3.17) we see that the uniform charge density which contributes to this divergence is the same as that of the seed geodesic. Therefore we regulate the charges by simply subtracting this charge density. Since $E=-S$ from (3.33) we see that we must define the following regulated charges

$$
\hat{E}=E+2 \hat{\lambda}\left(r_{+}+r_{-}\right) a_{0} b_{0} J L, \quad \hat{S}=S-2 \hat{\lambda}\left(r_{+}+r_{-}\right) a_{0} b_{0} J L .
$$

In terms of these regulated charges we obtain the following dispersion relation

$$
\hat{E}-\hat{S}=\hat{\lambda}\left(r_{+}+r_{-}\right) \frac{4 a_{0} b_{0}|\sin \theta|}{r} .
$$

The dispersion relation of these classical solutions resembles that of the giant magnon.

For the case $r=0$, the solution reduces to a geodesic but with different conserved charges. Substituting $r=0$ in (3.21) and using the result in the equations (3.25) we obtain the following geodesic

$$
\begin{array}{ll}
u^{\prime}=a_{0} \cos (\theta+J \tau), & v^{\prime}=a_{0} \sin (\theta+J \tau), \\
x^{\prime}=-b_{0} \sin (\theta-J \tau), & y^{\prime}=\cos (\theta-J \tau) .
\end{array}
$$


Evaluating the global charges for this case we obtain

$$
E+S=0, \quad E-S=-4 \pi \hat{\lambda}\left(r_{+}+r_{-}\right) a_{0} b_{0} \cos (2 \theta) .
$$

We now describe the snapshot of the string at a given value of the worldsheet time $\tau$. From the expression for the coordinates given in (3.25) we see that in general they acquire large values when the following equality is satisfied

$$
e^{-J_{1}}=\frac{b_{0}}{a_{0}} .
$$

Using the expression for $J_{1}$ in (3.21) we see that given a particular value of $\tau$, the equality in (3.40) will be satisfied at one point say $\sigma^{*}$. From the relations between the embedding coordinates $u, v, x, y$ and the BTZ coordinates $r, \theta, t$ given in (2.8) we see that at $\sigma^{*}$ the string possibly is at the boundary of the BTZ geometry. As discussed before the end points of the string move on time like geodesics. Thus at a given value of $\tau$ is pinned at the boundary and the end points lie on time like geodesics. An estimate of the worldsheet time at which the string falls into the horizon is given by

$$
\tau_{\text {fall }}=\operatorname{Min}\left(\frac{1}{J}\left|\tan ^{-1} \frac{b_{0}}{a_{0}}+\theta\right|, \frac{1}{J}\left|\tan ^{-1} \frac{b_{0}}{a_{0}}-\theta\right|\right),
$$

where the Min refers to the minimum of the quantities in the bracket. This estimate is obtained from the time at which the end points reach the horizon. We now plot the snapshot of the solution for some typical values of the parameters in the BTZ geometry. In figure 1 we have plotted the radial position of the solution (3.25) against the worldsheet $\sigma$ coordinate for the following set of parameters.

$$
b_{0}=\frac{0.9}{\sqrt{0.19}}, \quad r_{+}=10, \quad r_{-}=9, \quad J=\frac{0.8 \pi}{2}, \quad \theta=\frac{0.9 \pi}{2}, \quad r=1 .
$$

The above choice of $b_{0}$ gives $b_{0} / a_{0}=0.9$. Note that $r$ and $\phi$ reach asymptotic values at large values at $\sigma^{*}$ as predicted by the solution. The string is completely outside the horizon which is at $r_{+}=10$ for worldsheet time $\tau=1$. As $\tau$ increases we see that the end points cross the horizon and the point which is pinned at the boundary falls in. In figure 2 we have plotted the projection of the snapshot in the $r-\phi$ plane for $\tau=1$, the range of $\sigma$ chosen for the plot is from -5 to 5 . The two end points near the horizon are the ends of the strings which move on time like geodesics. The strings extends along the almost parallel lines which meet at the boundary of BTZ. Figure 3 shows the string configuration at $\tau=1$ in the 3 dimensional BTZ spacetime.

\section{Dressing space like geodesics}

Space like geodesics in the BTZ background play an important role in the framework of $A d S_{3} / C F T_{2}$. The regularized length of the space like geodesic connecting two equal time points on the boundary is a useful WKB estimate of the correlator of operators of large dimension operators inserted at these points [16-20]. Their lengths are also used to 

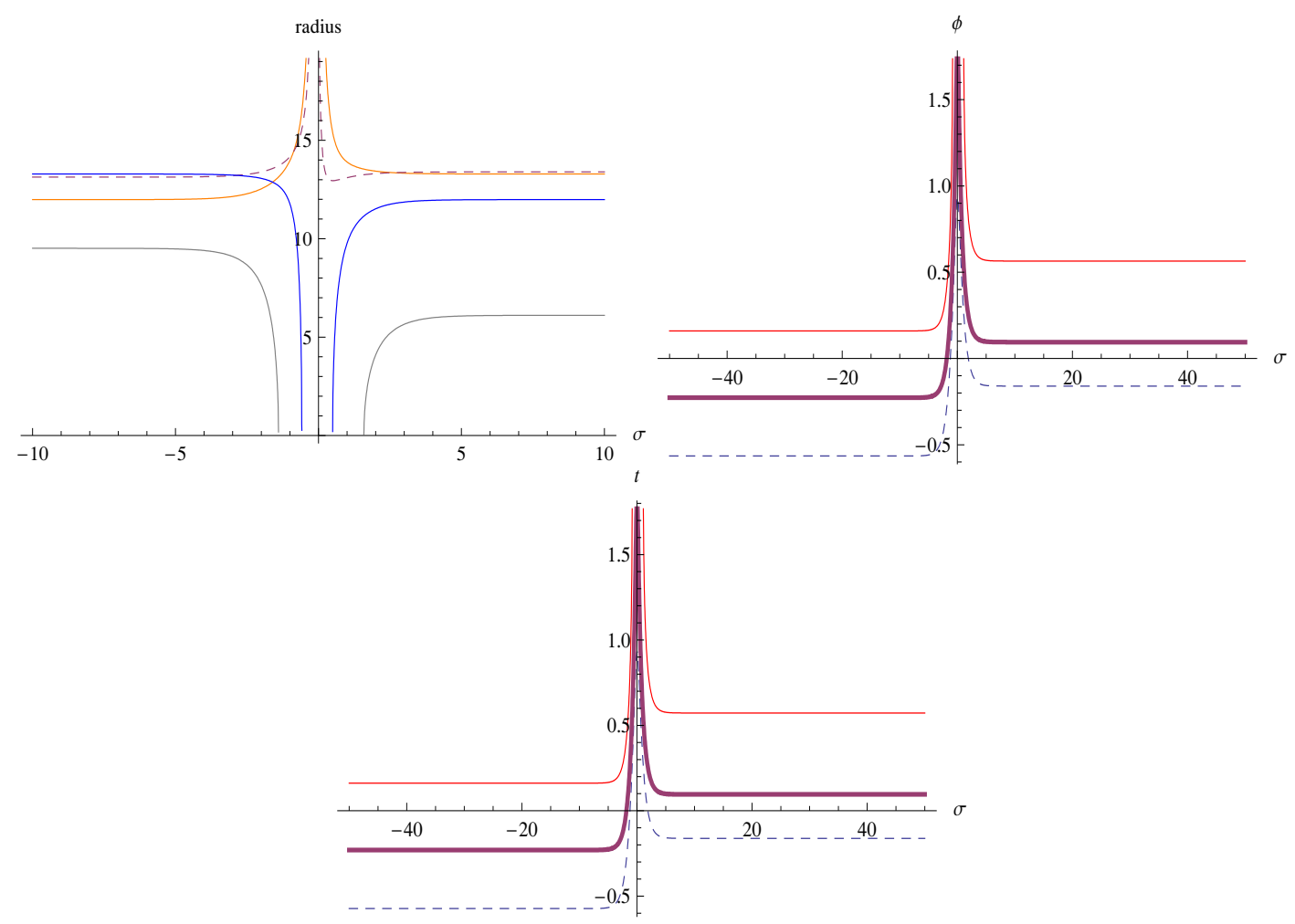

Figure 1. Orange $(\tau=1.0)$, dashed $(\tau=1.2)$, thick $(\tau=1.5)$, grey $(\tau=2.0)$.

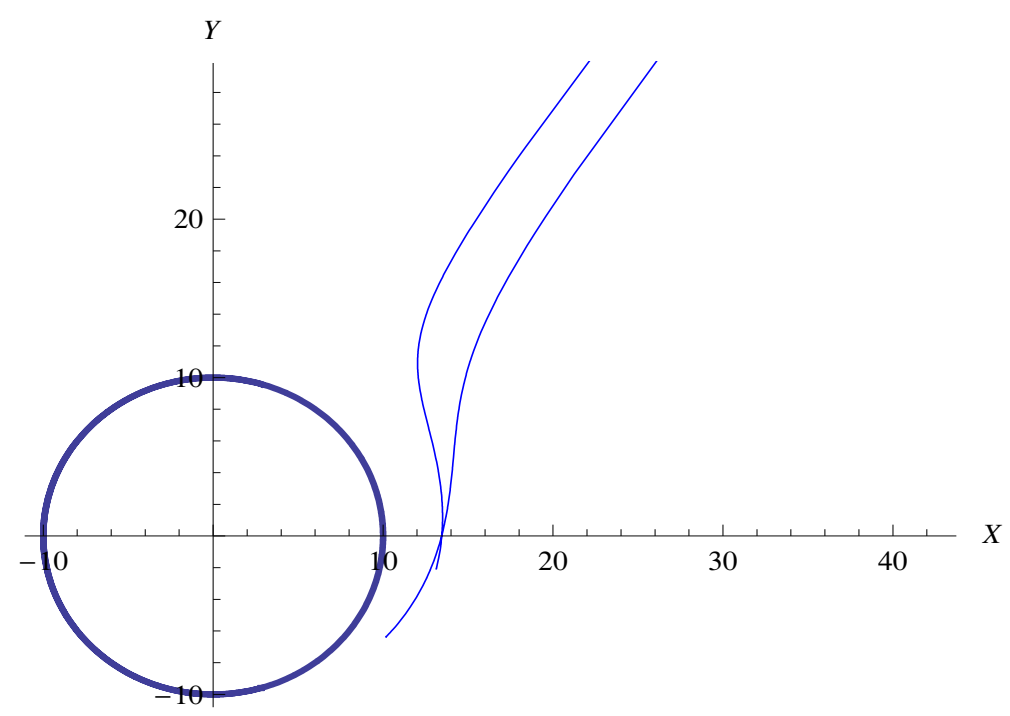

Figure 2. String in $r-\phi$ plane.

determine Wilson loops and entanglement entropy in the boundary theory [21, 28]. In the $\mathrm{SL}(2, R)$ WZW model they played an important role in generating new solutions using spectral flow [29]. In this section we study the solutions to the sigma model in the BTZ background obtained by dressing space like geodesics. Space like geodesics which ends on 


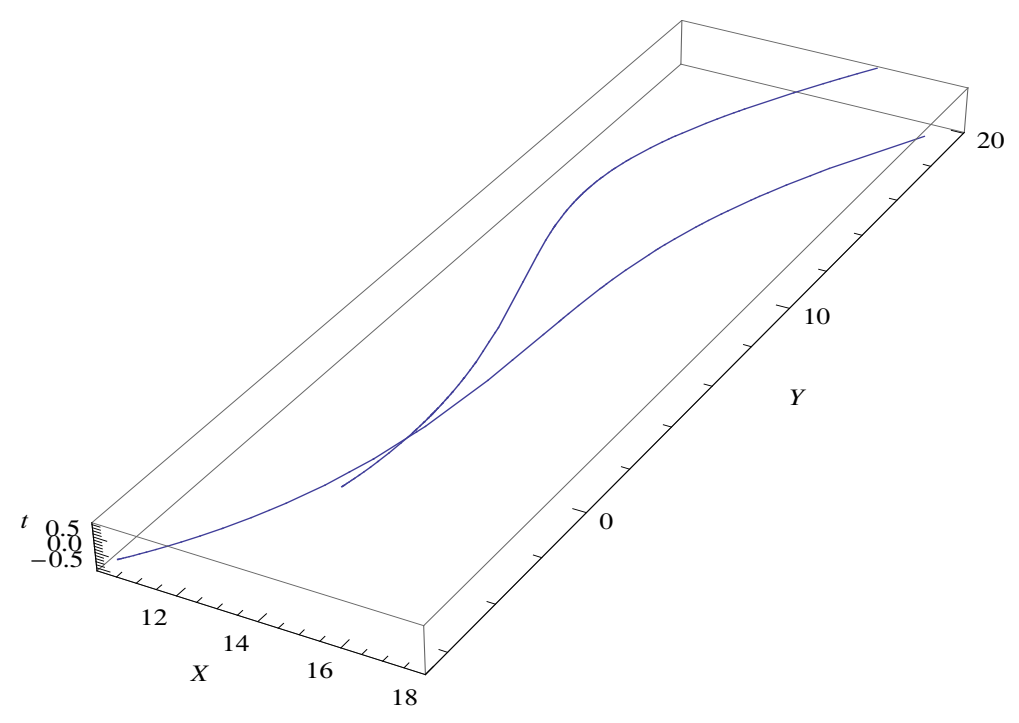

Figure 3. String in 3d BTZ background.

two points of the boundary never penetrate the horizon of the black hole. Starting at a point in the boundary they reach a minimum distance and before the turn back to the boundary. An interesting result we obtain is that the minimal surfaces obtained by dressing these space like geodesics can penetrate the horizon while being pinned on some curve at the boundary. Thus these can possibly be good probes of the physics at the horizon.

For the time like geodesics the mass of the particle was identified as the momentum $J$ of the circle in the $B T Z \times S^{1}$ sigma model given in (2.9). For the space like geodesics the equation of motion remains the same however the Virasoro constraints are changed. They are given by

$$
\dot{\gamma}^{2}+\frac{c_{1}^{2}}{\cosh ^{2} \gamma}-\frac{c_{2}^{2}}{\sinh ^{2} \gamma}-J^{2}=0
$$

Comparing this constraint with that corresponding to the time like case given in (3.2) we see that for the space like geodesics we need to replace $J^{2} \rightarrow-J^{2}$. We are now interested in geodesics that can originate from the boundary and reach back to the boundary. For this let us examine the potential for the space like geodesics. It is given by

$$
V(\gamma)=\frac{c_{1}^{2}}{a^{2}}-\frac{c_{2}^{2}}{b^{2}}-J^{2}
$$

For definiteness let us assume $c_{1}, c_{2}, J \geq 0$. Then the maximum of the potential occurs at

$$
\sinh \gamma=\sqrt{\frac{c_{2}}{c_{1}-c_{2}}} .
$$

This maximum exists for $c_{1}>c_{2}$. For the geodesic to turn back to the boundary we need the value at this maximum to be greater than zero. This is achieved by the following condition

$$
c_{1}-c_{2}>J .
$$




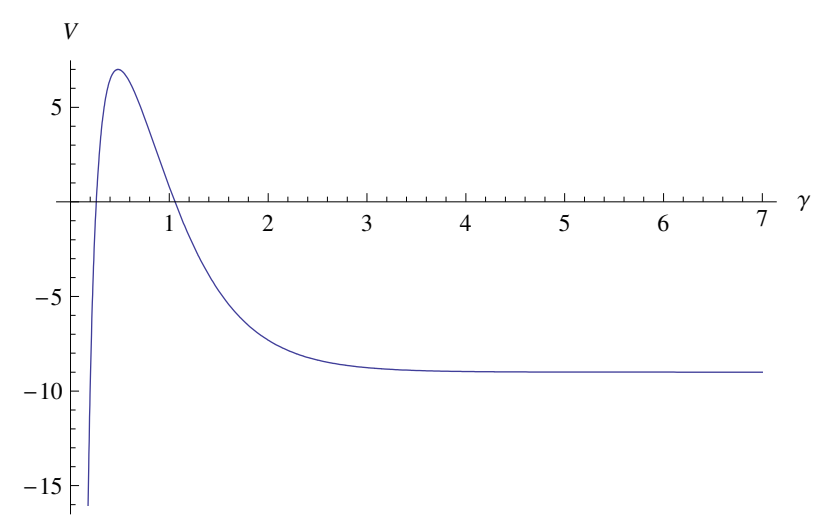

Figure 4. $\mathrm{V}(\gamma)$ vs $\gamma$ plot for $c_{1}=5, c_{2}=1, J=3$.

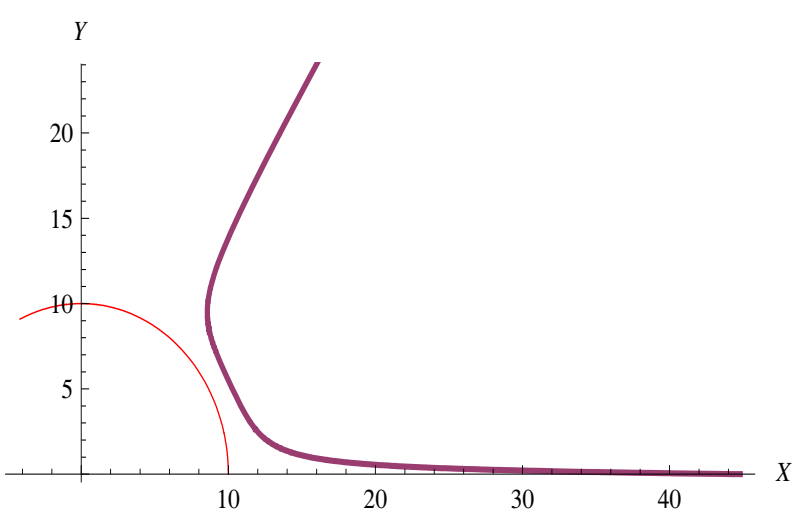

Figure 5. Trajectory of the tachyon for $c_{1}=5, c_{2}=1, J=3, r_{+}=10, r_{-}=9$ in $r-\phi$ plane of BTZ.

Once these conditions are satisfied it is clear that a geodesic starting at the boundary will return back to the boundary. It will never reach the horizon which is at $\gamma=0$. An example of values which satisfy these conditions are $c_{1}=5, c_{2}=1, J=3$. The potential is plotted in figure 4. A trajectory of a space like geodesic originating close to the boundary and going back to the boundary after hitting the hump located at

$$
\gamma_{c}=\sinh ^{-1}\left(\sqrt{\frac{c_{2}}{c_{2}-c_{1}}}\right)=0.4812
$$

is plotted in the $r-\phi$ plane in figure 5 .

It is clear from the generic shape of the potential for the condition $c_{1}-c_{2}>J$, that the space like geodesic never reaches the horizon. We will show that upon dressing these space like geodesics we will obtain classical string solutions that can penetrate the horizon while being pinned on the boundary at two points. The reason this is possible is perhaps due to the fact that the worldsheet in this case has Minkowski signature unlike the situation discussed in [28] where is was shown that Euclidean minimal surfaces pinned at the boundary do not penetrate horizons. We will also show that there is a domain of parameter space in which these solutions are closed strings. Just as space like geodesics 
which originate and end at the boundary can be used as probes of horizon physics it is plausible that these solutions can give information of the physics of the horizon.

To solve for the trajectory of a general space like geodesic we again use the ansatz given in (3.1). The initial conditions are given by

$$
a(0)=a_{0}, \quad b(0)=b_{0}, \quad f(0)=0, \quad b(0)=0, \quad \dot{\gamma}(0)=-\sqrt{J^{2}+\frac{c_{2}^{2}}{b_{0}^{2}}-\frac{c_{1}^{2}}{a_{0}^{2}}} .
$$

We start at some large $\gamma_{0}$ outside the maxima of the potential with radially inward velocity. The solution to the monodromy equations given in (3.7) can be obtained by following the similar procedure adopted for time like geodesics. The results are

$$
\Psi_{s}=\left(\begin{array}{ll}
\Psi_{s(11)} & \Psi_{s(12)} \\
\Psi_{s(21)} & \Psi_{s(22)}
\end{array}\right)
$$

where

$$
\begin{aligned}
& \Psi_{s(11)}=a_{0} \cosh [\vartheta]-\frac{b_{0} \sqrt{J^{2}+\frac{c_{2}^{2}}{b_{0}^{2}}-\frac{c_{1}^{2}}{a_{0}^{2}}}}{J} \sinh [\vartheta]+\frac{c_{1}}{a_{0} J} \sinh [\vartheta], \\
& \Psi_{s(12)}=b_{0} \cosh [\vartheta]-\frac{a_{0} \sqrt{J^{2}+\frac{c_{2}^{2}}{b_{0}^{2}}-\frac{c_{1}^{2}}{a_{0}^{2}}}}{J} \sinh [\vartheta]+\frac{c_{2}}{b_{0} J} \sinh [\vartheta], \\
& \Psi_{s(21)}=b_{0} \cosh [\vartheta]-\frac{a_{0} \sqrt{J^{2}+\frac{c_{2}^{2}}{b_{0}^{2}}-\frac{c_{1}^{2}}{a_{0}^{2}}}}{J} \sinh [\vartheta]-\frac{c_{2}}{b_{0} J} \sinh [\vartheta], \\
& \Psi_{s(22)}=a_{0} \cosh [\vartheta]-\frac{b_{0} \sqrt{J^{2}+\frac{c_{2}^{2}}{b_{0}^{2}}-\frac{c_{1}^{2}}{a_{0}^{2}}}}{J} \sinh [\vartheta]-\frac{c_{1}}{a_{0} J} \sinh [\vartheta],
\end{aligned}
$$

and $\vartheta$ and $J$ are defined in (3.11). Note that this solution satisfies the condition

$$
\Psi_{s}(0)=g,
$$

where $g$ refers to the trajectory of the space like geodesic with the initial conditions given in (4.6). To obtain this form of the monodromy matrix we have used the Virasoro constraint given in (4.1). Using the transformation given in (3.13) the solution to the $\mathrm{SU}(1,1)$ monodromy equations can be constructed. To write this solution in a convenient form we define

$$
c_{1}=n_{1} a_{0} J, \quad c_{2}=n_{2} b_{0} J,
$$

where $n_{1}$ and $n_{2}$ are arbitrary parameters. The solution to the $\mathrm{SU}(1,1)$ monodromy equations in (2.20) is then given by

$$
\begin{aligned}
& \Psi_{11}=\cosh \gamma_{0} \cosh \vartheta-c \sinh \left(\gamma_{0}\right) \sinh \vartheta-i n_{2} \sinh \vartheta, \\
& \Psi_{12}=n_{1} \sinh \vartheta+i\left(\sinh \gamma_{0} \cosh \vartheta-c \cosh \gamma_{0} \sinh \vartheta\right), \\
& \Psi_{21}=n_{1} \sinh \vartheta-i\left(\sinh \gamma_{0} \cosh \vartheta-c \cosh \gamma_{0} \sinh \vartheta\right), \\
& \Psi_{22}=\cosh \gamma_{0} \cosh \vartheta-c \sinh \left(\gamma_{0}\right) \sinh \vartheta+i n_{2} \sinh \vartheta,
\end{aligned}
$$


where

$$
c=\sqrt{1-n_{1}^{2}+n_{2}^{2}}
$$

We now proceed as in the case of the time like geodesics and construct the dressing factor given in (2.27) with the constant vector $e=\left(\begin{array}{l}1 \\ 0\end{array}\right)$. The dressed solution in the $\mathrm{SL}(2, R)$ sigma model is then obtained by using (3.15). This results in the following solution

$$
\begin{aligned}
u^{\prime}= & u \cos (\theta)+ \\
& \sin (\theta)\left[-\sinh \left(\gamma_{0}\right)\left(e^{\gamma^{\prime}} \sinh (J \tau-2 \alpha)+e^{\tilde{\gamma}} c \cosh (J \tau-2 \alpha)\right)+\right. \\
& \left.\left(c \sinh \left(\gamma_{0}\right) \cosh (J \tau)-\sinh (J \tau) \cosh \left(\gamma_{0}\right)\right)\left(e^{\tilde{\gamma}} \cos (2 \beta)+\sin (2 \beta)\right)\right] \\
& \times(\cos (2 \beta)-\exp (\tilde{\gamma}) \sin (2 \beta))^{-1}, \\
v^{\prime}= & v \cos (\theta) \\
& +\sin (\theta)\left[\cosh (J \tau-2 \alpha)\left(\cosh \left(\gamma_{0}\right)+n_{2} e^{\tilde{\gamma}}\right)+c \sinh \left(\gamma_{0}\right) \sinh (J \tau-2 \alpha)\right. \\
& \left.-n_{2} \cosh (J \tau)\left(e^{\tilde{\gamma}} \cos (2 \beta)+\sin (2 \beta)\right)\right](\cos (2 \beta)-\exp (\tilde{\gamma}) \sin (2 \beta))^{-1}, \\
x^{\prime}= & x \cos (\theta) \\
& +\sin (\theta)\left[\cosh (J \tau-2 \alpha)\left(n_{1} e^{\tilde{\gamma}}-\sinh \left(\gamma_{0}\right)\right)-c \cosh \left(\gamma_{0}\right) \sinh (J \tau-2 \alpha)\right. \\
& \left.-n_{1} \cosh (J \tau)\left(e^{\tilde{\gamma}} \cos (2 \beta)+\sin (2 \beta)\right)\right](\cos (2 \beta)-\exp (\tilde{\gamma}) \sin (2 \beta))^{-1}, \\
y^{\prime}= & y \cos (\theta)+ \\
& \sin (\theta)\left[-\cosh \left(\gamma_{0}\right)\left(e^{\gamma^{\prime}} \sinh (J \tau-2 \alpha)+e^{\tilde{\gamma}} c \cosh (J \tau-2 \alpha)\right)+\right. \\
& \left.\left(c \cosh \left(\gamma_{0}\right) \cosh (J \tau)-\sinh (J \tau) \sinh \left(\gamma_{0}\right)\right)\left(e^{\tilde{\gamma}} \cos (2 \beta)+\sin (2 \beta)\right)\right] \\
& \times(\cos (2 \beta)-\exp (\tilde{\gamma}) \sin (2 \beta))^{-1},
\end{aligned}
$$

where

$$
e^{\tilde{\gamma}}=n_{2} \cosh \left(\gamma_{0}\right)+n_{1} \sinh \left(\gamma_{0}\right), \quad e^{\gamma^{\prime}}=n_{1} \cosh \left(\gamma_{0}\right)+n_{2} \sinh \left(\gamma_{0}\right),
$$

and

$$
\alpha=\frac{\tau\left(1-r^{2} \cos (2 \theta)\right)+\sigma \cos (\theta)\left(1-r^{2}\right)}{1+r^{4}-2 r^{2} \cos (2 \theta)} J, \quad \beta=\frac{\tau r^{2} \sin (2 \theta)+r \sigma \sin (\theta)\left(1+r^{2}\right)}{1+r^{4}-2 r^{2} \cos (2 \theta)} J
$$

Note that $\alpha=J_{2} / 2$ and $\beta=J_{1} / 2$ as defined in (3.21). We have also explicitly verified that these solutions satisfy the equations of motion as well as the following Virasoro constraints

$$
-\partial_{ \pm}\left(u^{\prime}+x^{\prime}\right) \partial_{ \pm}\left(u^{\prime}-x^{\prime}\right)+\partial_{ \pm}\left(y^{\prime}+v^{\prime}\right) \partial_{ \pm}\left(y^{\prime}-v^{\prime}\right)=J^{2}
$$

Note that compared to the Virasoro constraints of solutions obtained by dressing time like geodesics in (3.26) we have $J^{2} \rightarrow-J^{2}$. This is expected from the general proof that the Virasoro constraints of the original solution are preserved by the dressing method. Since these are not the Virasoro constraints obeyed by a physical string these solutions are best thought of as minimal surfaces in the BTZ background. 


\subsection{Closed string solutions}

The solutions given in the equations (4.13) are in general open strings and are complicated. To get more insight into them we discuss the interesting situation when the solutions given in (4.13) become periodic in $\sigma$. Two special cases for the above solutions are $r=1$ and $\theta=\pi / 2$. Note that for either of these two cases, $\alpha$ in (4.14) loses its $\sigma$ dependence. For these cases the solution given in (4.13) depends on $\sigma$ through the trigonometric functions of the angle $\beta$ which in turn depends linearly on $\sigma$. Thus the solutions are periodic in $\sigma$ with the period determined by the coefficient multiplying $\sigma$ in $\beta$ given in (4.14). Thus the periodicity $\sigma_{p}$ of the closed string is given by

$$
\sigma_{p}=\frac{\pi\left(1+r^{4}-2 r^{2} \cos (2 \theta)\right)}{J r \sin (\theta)\left(1+r^{2}\right)} .
$$

Closed strings with $\boldsymbol{r}=\mathbf{1}$, arbitrary $\boldsymbol{\theta}$ We will discuss the case $r=1$ with arbitrary $\theta$ in some detail. These solutions have $\sigma$ dependance through the periodic trigonometric functions. The solutions are now closed strings with period

$$
\sigma_{p}=\frac{2 \pi}{J} \sin \theta .
$$

We will now evaluate the global charges for these solutions. From the same analysis leading up to equation (3.33) we obtain the following expression for the charge $E+S$

$$
\begin{aligned}
\frac{2(E+S)}{\hat{\lambda}\left(r_{+}-r_{-}\right)} & =\int_{0}^{2 \pi \sigma_{p}} d \sigma \operatorname{Tr}\left(\partial_{0} \hat{g} \hat{g}^{-1} \sigma^{1}\right)-(\lambda-\bar{\lambda}) \operatorname{Tr}\left(\left(P\left(\tau, 2 \pi \sigma_{p}\right)-P(\tau, 0)\right) \sigma^{1}\right), \\
& =\int_{0}^{2 \pi \sigma_{p}} d \sigma \operatorname{Tr}\left(\partial_{0} g g^{-1} \sigma^{3}\right), \\
& =\frac{4 \pi\left(c_{1}-c_{2}\right) \sin \theta}{J} .
\end{aligned}
$$

To obtain the second line we have used the periodicity property of the solution and also the relation between the $\mathrm{SL}(2, R)$ group element $g$ and the $\mathrm{SU}(1,1)$ group element denoted by $\hat{g}$. We have also verified the last line of the equation by explicitly evaluating this charge on the dressed solution $g^{\prime}$ given in (4.13). Thus we obtain the simple result that the charge $E+S$ of the dressed solution reduces to the charge density of the seed geodesic multiplied by the periodicity of the string. For the charge $E-S$ we obtain a similar result though we do not have a simple proof. Explicitly evaluating the charge density $E-S$ for the solution (4.13) we obtain

$$
\frac{2(E-S)}{\hat{\lambda}\left(r_{+}-r_{-}\right)}=\frac{4 \pi\left(c_{1}+c_{2}\right) \sin \theta}{J}
$$

Here again we find that the charge is equal to the charge density corresponding to the seed geodesic multiplied by the length of the closed string. ${ }^{2}$ Again the equations for the charge

\footnotetext{
${ }^{2}$ We have also verified that the property that the global charges are equal to the charged density of the corresponding seed geodesic multiplied by the length of the closed string remains true for the situation with $\theta=\frac{\pi}{2}$ and arbitrary $r$.
} 
in (4.18) and (4.19) closely resemble that of giant magnon dispersion relations. Note that in this analysis we have chosen $\sin \theta>0$, whereas for $\sin \theta<0$ the expressions in (4.18) and (4.19) are similar with $\sin \theta$ replaced by $|\sin \theta|$.

We now qualitatively describe the motion of the string in the BTZ background. From the solution given in (4.13) we see that the coordinates $u^{\prime}, v^{\prime}, x^{\prime}, y^{\prime}$ assume large values when

$$
\cot 2 \beta^{*}=e^{\tilde{\gamma}}
$$

which implies

$$
\beta_{ \pm}^{*}=\cot ^{-1}\left(e^{\tilde{\gamma}} \pm \sqrt{e^{2 \tilde{\gamma}}+1}\right) .
$$

From (4.14) we see that for $r=1, \beta$ is given by

$$
\beta=\frac{J}{2}\left(\frac{\tau \cos \theta+\sigma}{\sin \theta}\right) .
$$

Thus given $\tilde{\gamma}$, the equation (4.21) determines a line in the worldsheet coordinates at which the space time coordinates become large. From the relationship between the radial coordinate of the BTZ to the coordinates $u^{\prime}, v^{\prime}, x^{\prime}, y^{\prime}$ given in (2.8) we see that when the equation (4.21) holds it is possible that the minimal surface can reach the boundary of BTZ.

To describe the solution further we examine the solution at the following parameters

$$
\gamma_{0}=3, \quad r_{+}=10, \quad r_{-}=9, \quad J=3, \quad c_{1}=5, \quad c_{2}=1, \quad \theta=\frac{\pi}{4}, \quad r=1 .
$$

As before the outer and inner horizon radius of BTZ is chosen to be $r_{+}=10$ and $r_{-}=9$ respectively. Note that as discussed earlier and from figure 4 and figure 5 the seed space like geodesic for these set of parameters originates close to the boundary and bounces back to the boundary after reaching near the horizon. It does not penetrate the horizon. The periodicity of the string for this set of parameters is given by

$$
\sigma_{p}=\frac{\sqrt{2} \pi}{3} .
$$

The values of $\beta_{ \pm}^{*}$ are given by

$$
\beta_{+}^{*}=0.232484, \quad \beta_{-}^{*}=-1.33831 .
$$

The curve on the world sheet for which the space time coordinates become large is given by

$$
\sigma_{ \pm}^{*}=\frac{\sqrt{2}}{3} \beta_{ \pm}^{*}-\frac{\tau}{\sqrt{2}} .
$$

Thus given a value of $\tau$, there are two values of $\sigma$ at which the space time coordinates are large. To visualize the minimal surface we plot its snapshot at various values of worldsheet time. The radial $r$, time $t$ and the angular coordinate $\phi$ are plotted with respect to the $\sigma$ coordinate at worldsheet times $\tau=.5,1.0,1.34$ in figure 6 . The cross section of the minimal surface in the $r-\phi$ plane is provided in figure 7 and figure 8 . The range of the 

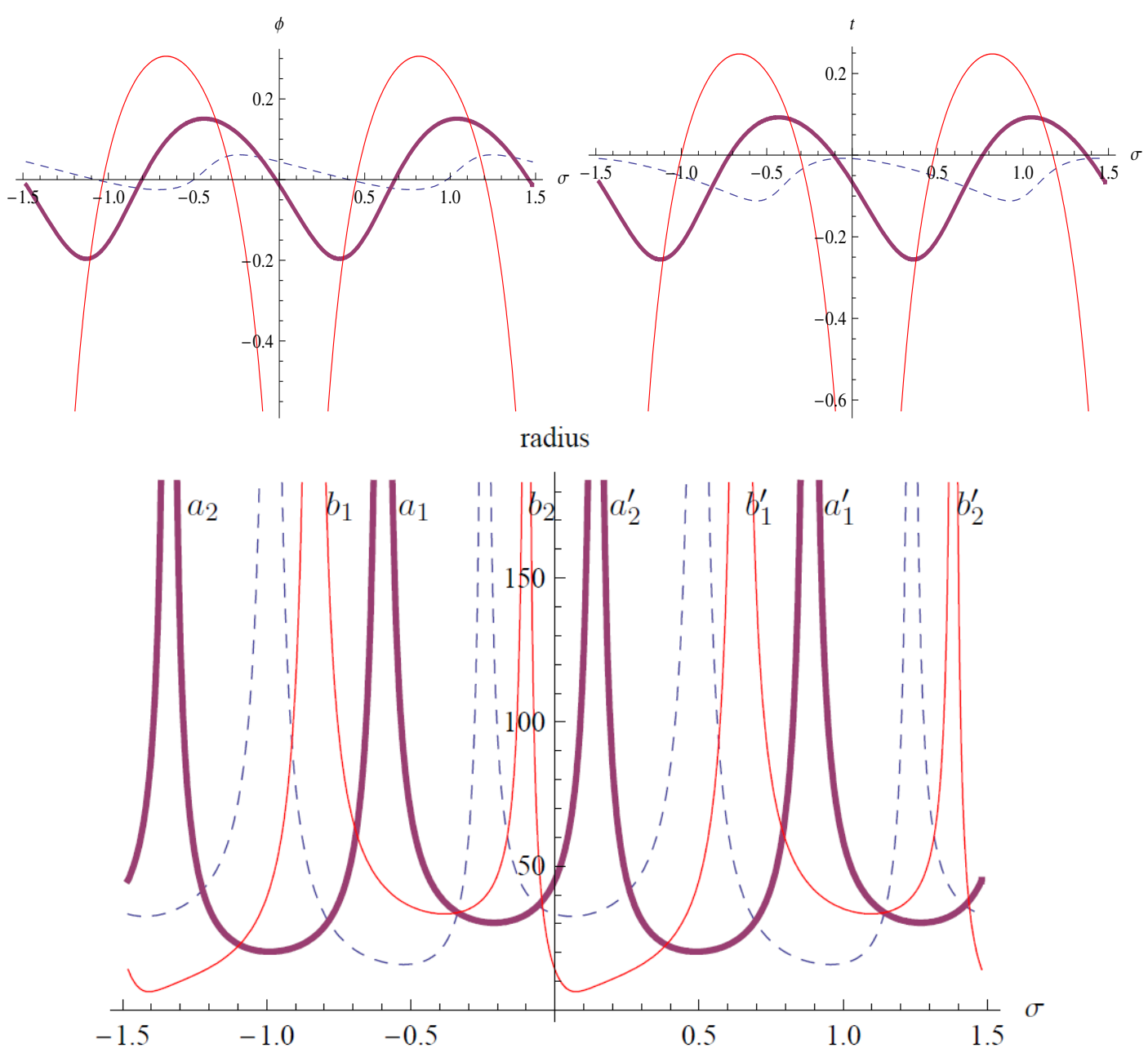

Figure 6. Dashed $(\tau=0.5)$, thick $(\tau=1.0)$, red $(\tau=1.34)$.

$\sigma$ axis runs from $-\sqrt{2} \pi / 3$ to $\sqrt{2} \pi / 3$ in figure 6 . The fact that the string is closed with period $\sqrt{2} \pi / 3$ is clearly visible in figure 6 . In figure 6 we have also marked the position at which the radial coordinate becomes infinite for the time $\tau=1.0,1.34$. For $\tau=1.0$, the value of $\sigma_{+}^{*}$ and its periodic image is marked by $a_{1}$ and $a_{1}^{\prime}$ and the value of $\sigma_{-}^{*}$ and its periodic image is marked by $a_{2}$ and $a_{2}^{\prime}$. Similarly for $\tau=1.34$ the value of $\sigma_{+}^{*}$ and its image is marked by $b_{1}$ and $b_{1}^{\prime}$, the value of $\sigma_{-}^{*}$ and its image is marked by $b_{2}$ and $b_{2}^{\prime}$. From the plots we see that the surface is pinned at the boundary on two curves and hangs into the bulk. As the worldsheet time is increased the surface crosses the horizon while being pinned at the boundary. Eventually the string reaches the singularity. We can also describe the two curves on the boundary at which the minimal surface is pinned. These curves are given by substituting the relations for $\sigma_{+}, \sigma_{-}$given in (4.26) into the expression for the time coordinate $t$ and the angular coordinate $\phi$ given in (2.8). Then one can obtain a parametric plot of $t$ and $\phi$ with respect to the parameter $t$. These two curves corresponding to $\beta_{+}^{*}$ and $\beta_{-}^{*}$ are given in figure 9 and figure 10 respectively. From the figures we see that the curves describe a time like on the boundary. 


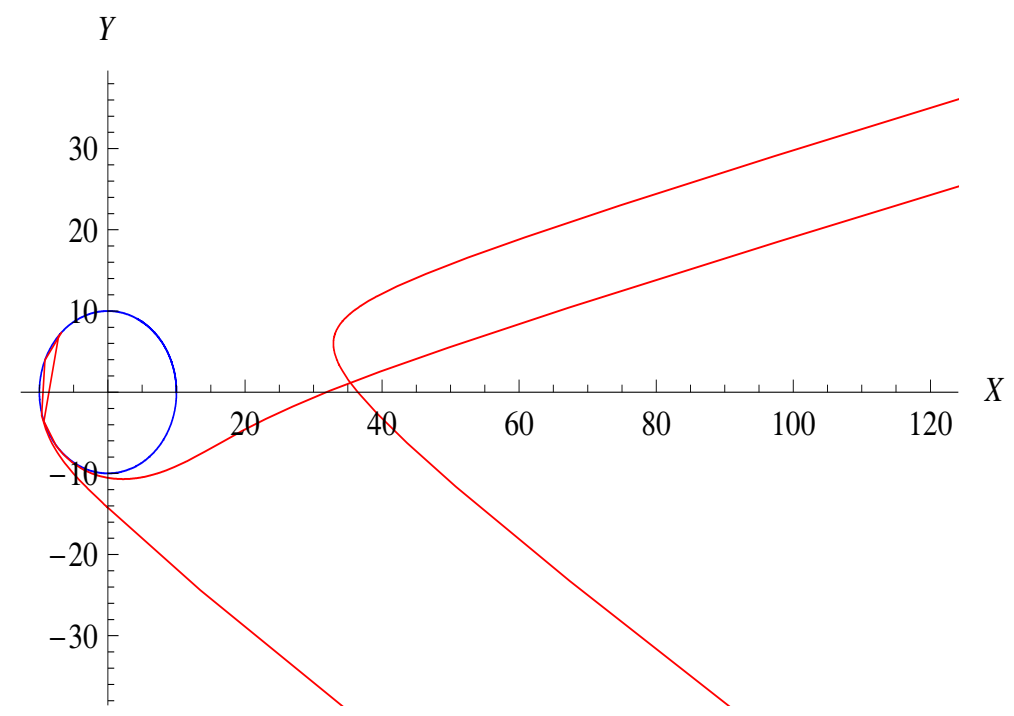

Figure 7. Spacelike strings in $r-\phi$ plane at $\tau=1.34$.

Since these solutions can be interpreted as minimal surfaces it is interesting to verify if their proper area given by the Nambu Goto Lagrangian is positive for all $\sigma$ for the worldsheet times of interest. ${ }^{3}$ The Nambu-Goto Lagrangian density is given by

$$
\left.\mathcal{L}_{N G}=\left[\operatorname{Tr}\left(g^{-1} g^{\prime} g^{-1} \dot{g}\right)\right)^{2}-\operatorname{Tr}\left(g^{-1} \dot{g} g^{-} \dot{g}\right) \operatorname{Tr}\left(g^{-1} g^{\prime} g^{-1} g^{\prime}\right)\right]^{1 / 2},
$$

where $g$ is given in terms of the embedding in (2.6). Here the superscripts prime and dot refer to derivatives with respect to $\sigma$ and worldsheet time $\tau$ respectively. We have verified that this Lagrangian density is positive for all $\tau=0.5$ to $\tau=1.34$. For a sample of its profile we have plotted the Lagrangian density for $\tau=1.0$ with respect to the worldsheet $\sigma$ coordinate in figure 11.

\section{Giant gluons in BTZ}

The giant gluon solution found in [31, 32] is a solution of the Euclidean worldsheet equations of motion in $A d S_{3}$. This solution has played an important role in evaluating the gluon scattering amplitudes in AdS. It is interesting to study its properties in the BTZ background. The embedding can be done in BTZ in two different ways consistent with the periodicities of the BTZ background.

Method 1. In this method the giant gluon solution can be written in terms of BTZ embedding coordinates given in (2.6) as follows

$$
\begin{array}{ll}
u=\cosh \sigma \cosh \tau, & v=\sinh \sigma \sinh \tau, \\
x=\sinh \sigma \cosh \tau, & y=\cosh \sigma \sinh \tau .
\end{array}
$$

\footnotetext{
${ }^{3}$ This check was suggested to us by Aninda Sinha.
} 


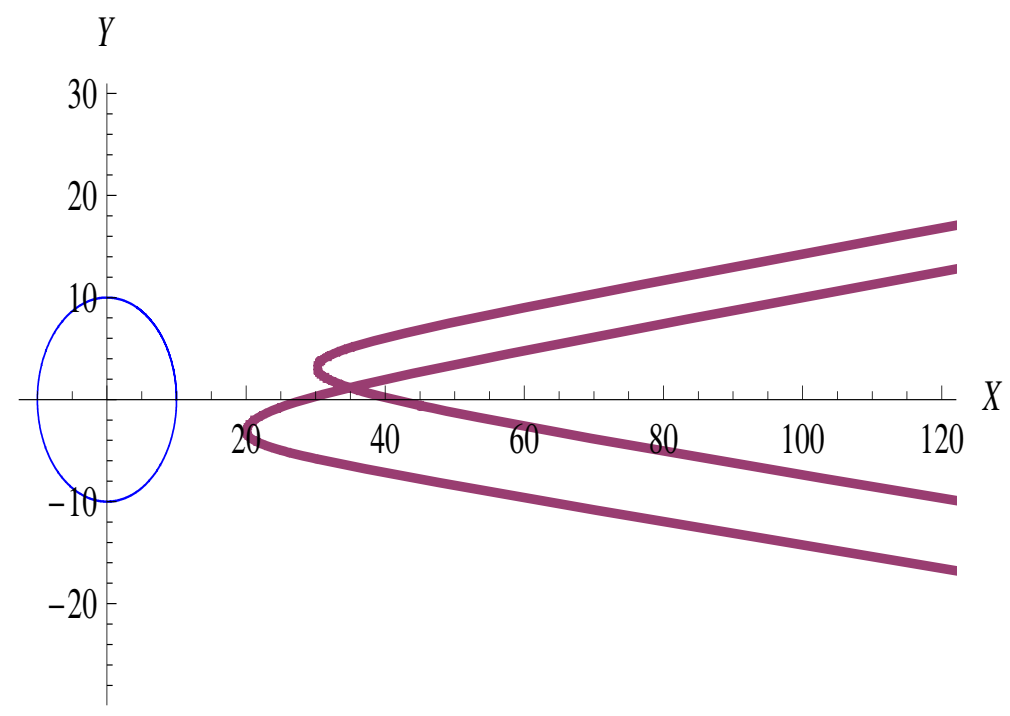

Figure 8. Spacelike strings in $r-\phi$ plane at $\tau=1.0$.

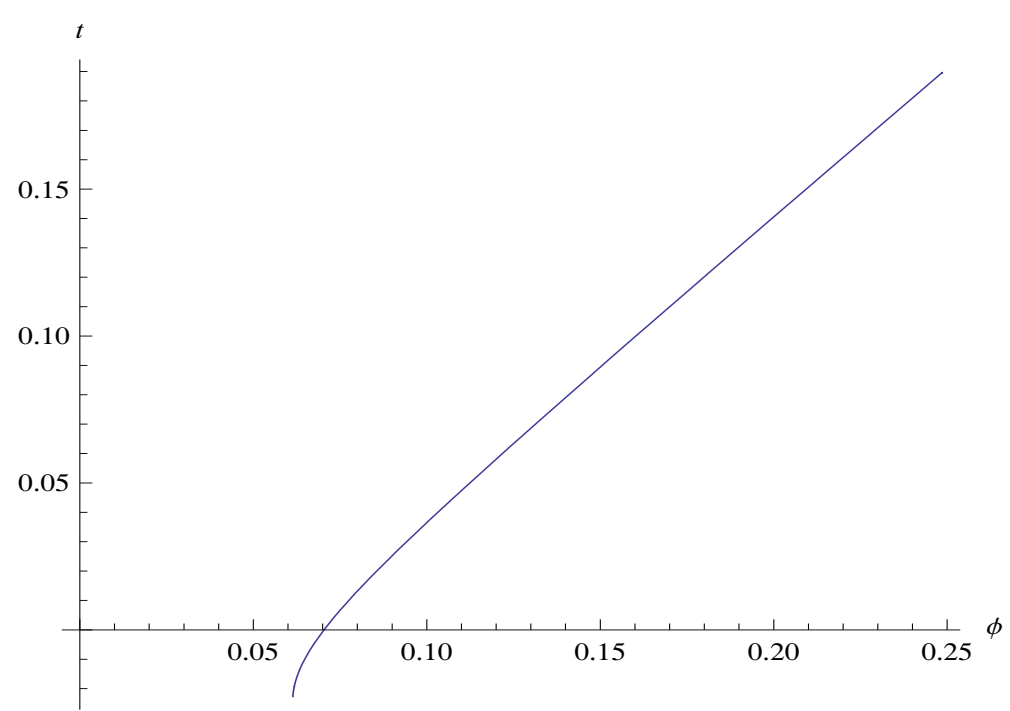

Figure 9. Propagation of the BTZ boundary point due to root $\beta_{1}$ from $\tau=0.5$ to 1.34 .

With the above choice the $\mathrm{SL}(2, R)$ group element becomes

$$
g=\left(\begin{array}{cc}
\cosh \tau e^{\sigma} & \sinh \tau e^{\sigma} \\
\sinh \tau e^{-\sigma} & \cosh \tau e^{-\sigma}
\end{array}\right) .
$$

The conserved left and right currents are

$$
\begin{array}{ll}
j_{\tau}=\partial_{\tau} g g^{-1}=\left(\begin{array}{cc}
0 & e^{2 \sigma} \\
e^{-2 \sigma} & 0
\end{array}\right), & j_{\sigma}=\partial_{\sigma} g g^{-1}=\left(\begin{array}{cc}
1 & 0 \\
0 & -1
\end{array}\right), \\
l_{\tau}=g^{-1} \partial_{\tau} g=\left(\begin{array}{ll}
0 & 1 \\
1 & 0
\end{array}\right), & l_{\sigma}=g^{-1} \partial_{\sigma} g=\left(\begin{array}{cc}
\cosh 2 \tau & \sinh 2 \tau \\
-\sinh 2 \tau & -\cosh 2 \tau
\end{array}\right) .
\end{array}
$$




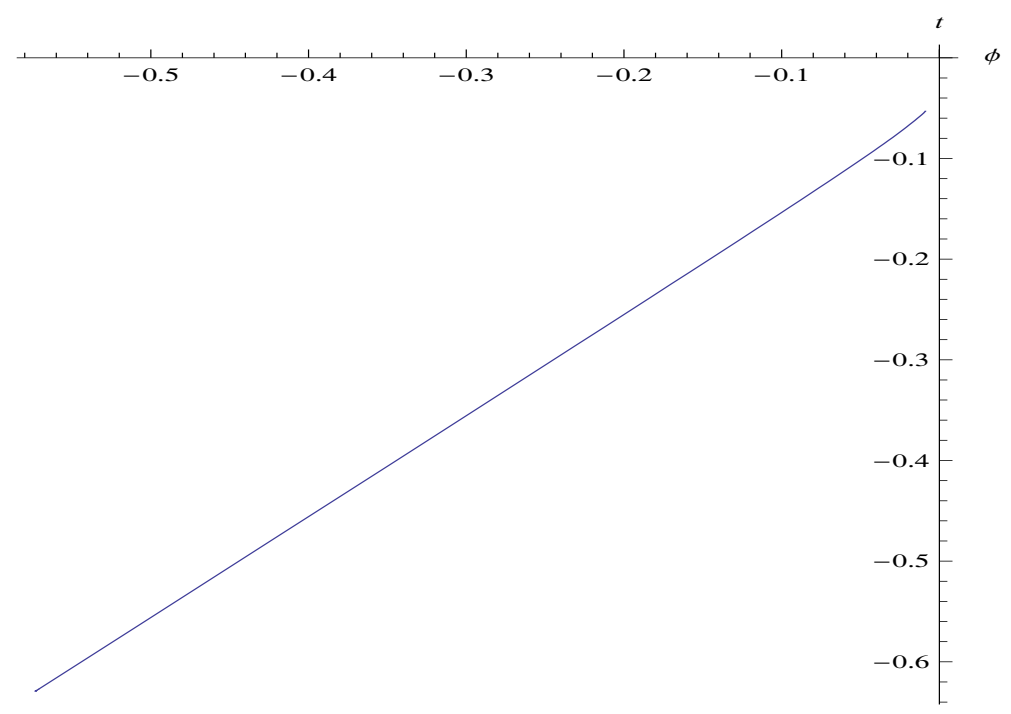

Figure 10. Propagation of the BTZ boundary point due to $\operatorname{root} \beta_{2}$ from $\tau=0.5$ to 1.34 .

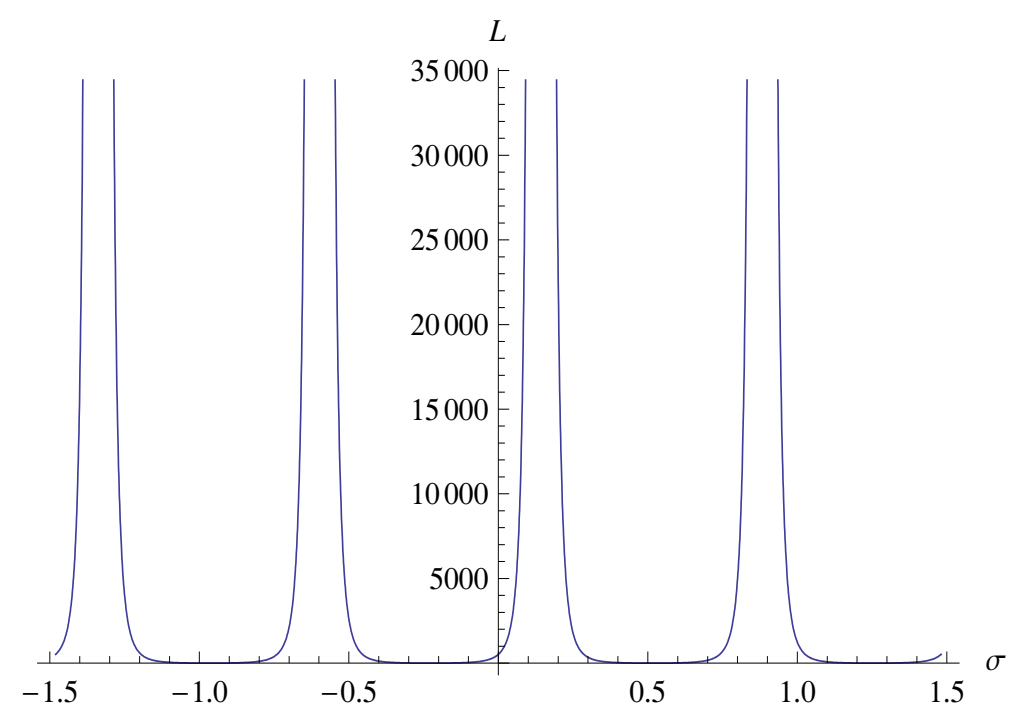

Figure 11. Worldsheet Lagrangian vs $\sigma$ plot for space like strings.

From these currents it can be seen that this solution satisfies the Euclidean worldsheet equations of motion trivially, i.e.

$$
\partial_{\tau} j_{\tau}+\partial_{\sigma} j_{\sigma}=0
$$

They also satisfy the Euclidean Virasoro constraints which are

$$
-\partial_{ \pm}(u+x) \partial_{ \pm}(u-x)+\partial_{ \pm}(y+v) \partial_{ \pm}(y-v)=0,
$$

where $\partial_{ \pm}=i \partial_{\tau} \pm \partial_{\sigma}$. The factor of $i$ in front of $\partial_{\tau}$ accounts for the fact that these solutions are Euclidean. The global charges $E$ and $S$ are zero for this configuration, since $\operatorname{Tr}\left(j_{\tau} \sigma_{3}\right)$ and $\operatorname{Tr}\left(l_{\tau} \sigma_{3}\right)$ vanish. 


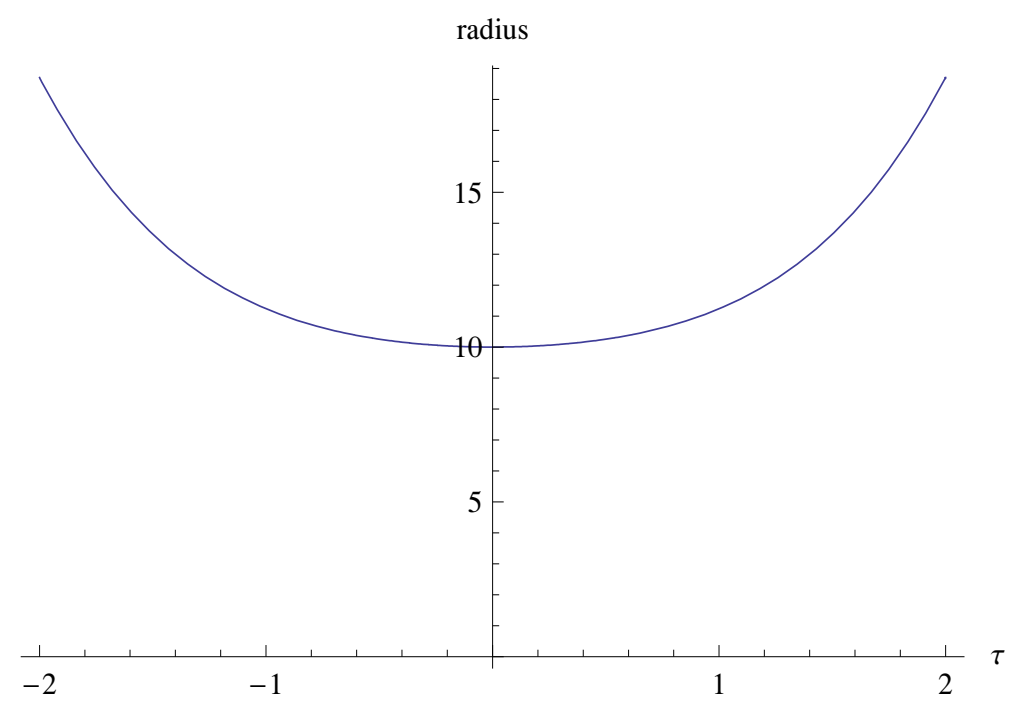

Figure 12. Plot of $r$ with respect to $\tau$.

Let us examine the behaviour of the solution in space time. In terms of the BTZ coordinates the solution has a rather simple form

$$
\begin{aligned}
& r=\sqrt{\left(r_{+}^{2}-r_{-}^{2}\right)\left(y^{2}-v^{2}\right)+r_{+}^{2}}=\sqrt{\left(r_{+}^{2}-r_{-}^{2}\right)\left(\sinh ^{2} \tau\right)+r_{+}^{2}}, \\
& \phi=\frac{r_{+} \log \left[\frac{u+x}{u-x}\right]+r_{-} \log \left[\frac{y+v}{y-v}\right]}{2\left(r_{+}^{2}-r_{-}^{2}\right)}=\frac{\sigma}{r_{+}-r_{-}}, \\
& t=\frac{r_{-} \log \left[\frac{u+x}{u-x}\right]+r_{+} \log \left[\frac{y+v}{y-v}\right]}{2\left(r_{+}^{2}-r_{-}^{2}\right)}=\frac{\sigma}{r_{+}-r_{-}} .
\end{aligned}
$$

From figure 12 we notice that $r$ is an even function of $\tau$ only and the minimum of $r$ occurs at $r_{+}$at $\tau=0$. The space time coordinates $t$ and $\phi$ are linear in $\sigma$. Thus a snapshot of the string at a given value of $\tau$ has a spiral shape as shown in figure 13. As $\tau$ increases or decreases from $\tau=0$, the radius of the spiral increases since $r$ is an even function of $\tau$. Since this embedding has trivial global charges $E, S$ it is degenerate with the BTZ state in the CFT at least with respect to these charges.

Method 2. The other possible configuration for the string in $B T Z$ is written by interchanging $x$ and $y$ in method 1 . This is given by

$$
\begin{array}{ll}
u=\cosh \sigma \cosh \tau, & v=\sinh \sigma \sinh \tau, \\
y=\sinh \sigma \cosh \tau, & x=\cosh \sigma \sinh \tau,
\end{array}
$$

and the $\mathrm{SL}(2, R)$ group reduces to

$$
g=\left(\begin{array}{cc}
\cosh \sigma e^{\tau} & \sinh \sigma e^{\tau} \\
\sinh \sigma e^{-\tau} & \cosh \sigma e^{-\tau}
\end{array}\right)
$$



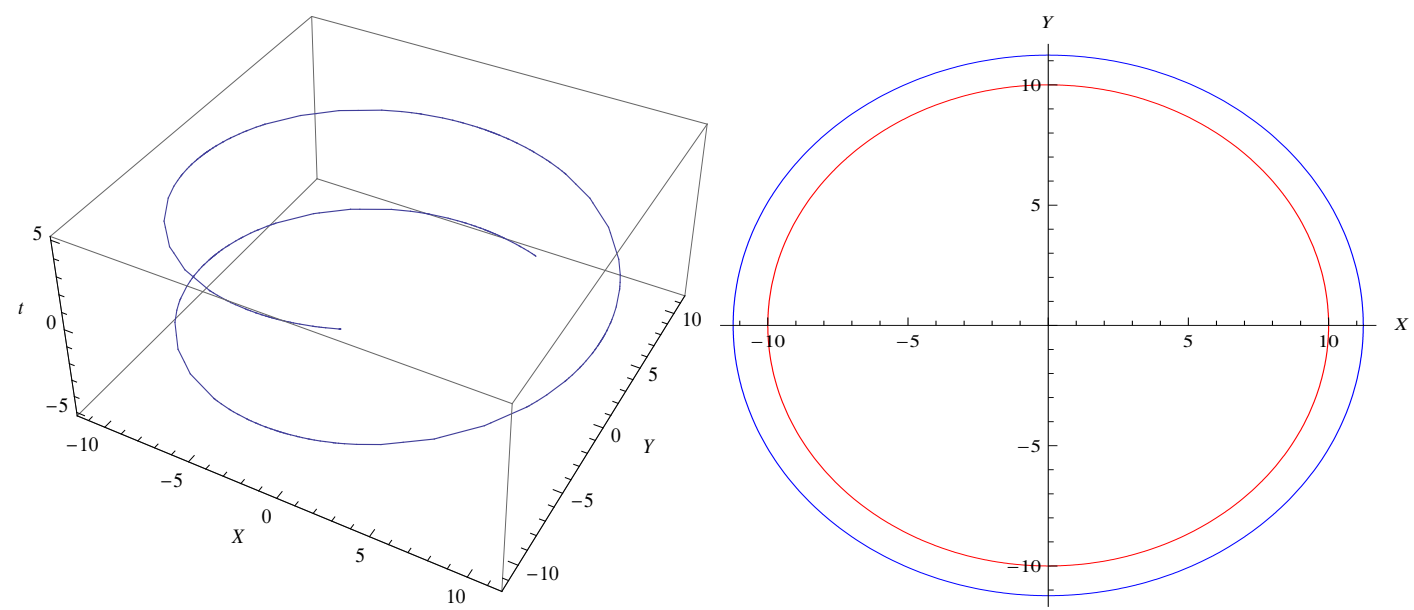

Figure 13. The $3 \mathrm{~d}$ plot shows a spiral string at $\tau=1$ and for $\sigma \in[-5,5]$ and the $2 \mathrm{~d}$ plot shows the projection of the spiral in $r-\phi$ plane. The red circle denotes the position of the horizon.

Note that this embedding is obtained from the one in method 1 by the interchange of $\sigma$ and $\tau$. The conserved left and right currents are

$$
\begin{array}{ll}
j_{\tau}=\partial_{\tau} g g^{-1}=\left(\begin{array}{cc}
1 & 0 \\
0 & -1
\end{array}\right), & j_{\sigma}=\partial_{\sigma} g=\left(\begin{array}{cc}
0 & e^{2 \tau} \\
e^{-2 \tau} & 0
\end{array}\right), \\
l_{\tau}=g^{-1} \partial_{\tau} g=\left(\begin{array}{cc}
\cosh 2 \sigma & \sinh 2 \sigma \\
\sinh 2 \sigma & -\cosh 2 \sigma
\end{array}\right), & l_{\sigma}=g^{-1} \partial_{\sigma} g=\left(\begin{array}{cc}
\cosh 2 \tau & \sinh 2 \tau \\
-\sinh 2 \tau & -\cosh 2 \tau
\end{array}\right) .
\end{array}
$$

This solution satisfies and Euclidean equations of motion and the corresponding Virasoro constraint given in (5.5). Unlike the case of the embedding in method 1, this solution has non zero values for $E$ and $S$. It is

$$
\begin{aligned}
E+S & =\frac{\hat{\lambda}}{2}\left(r_{+}-r_{-}\right) \int_{-\Lambda}^{\Lambda} d \sigma \operatorname{Tr}\left(j_{\tau} \sigma_{3}\right) \\
& =2 \Lambda \hat{\lambda}\left(r_{+}-r_{-}\right)
\end{aligned}
$$

and

$$
\begin{aligned}
E-S & =-\frac{\hat{\lambda}}{2}\left(r_{+}+r_{-}\right) \int_{-\Lambda}^{\Lambda} d \sigma \operatorname{Tr}\left(l_{\tau} \sigma_{3}\right) \\
& =-\frac{\hat{\lambda}}{2}\left(r_{+}+r_{-}\right) e^{2 \Lambda},
\end{aligned}
$$

where we have introduced the cut-off $\Lambda$. For large $\Lambda$ we can solve for $S$ and eliminate the cut-off to obtain the dispersion relation

$$
E+S \sim \hat{\lambda}\left(r_{+}-r_{-}\right) \ln \left(\frac{4 S}{\hat{\lambda}\left(r_{+}+r_{-}\right)}\right) .
$$

These dispersion relations are similar to that seen in the giant gluon solution. 


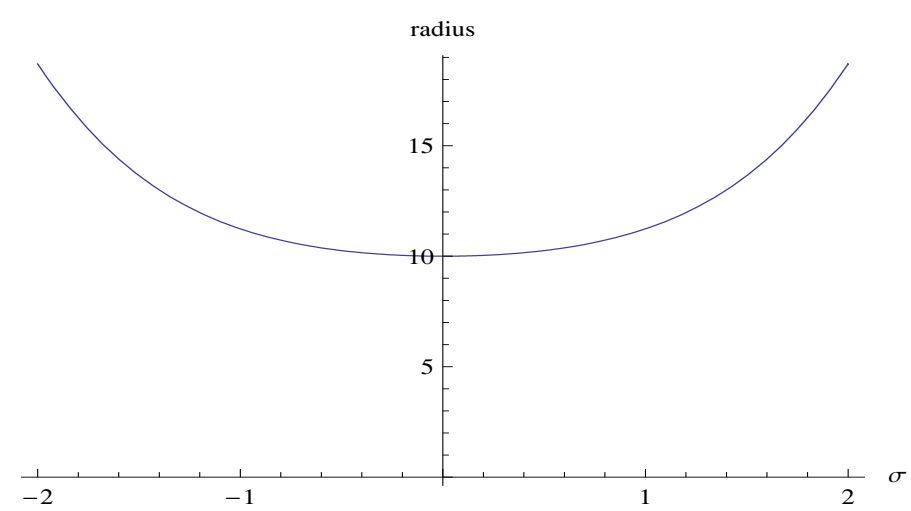

Figure 14. Variation of $r$ with respect to $\sigma$.
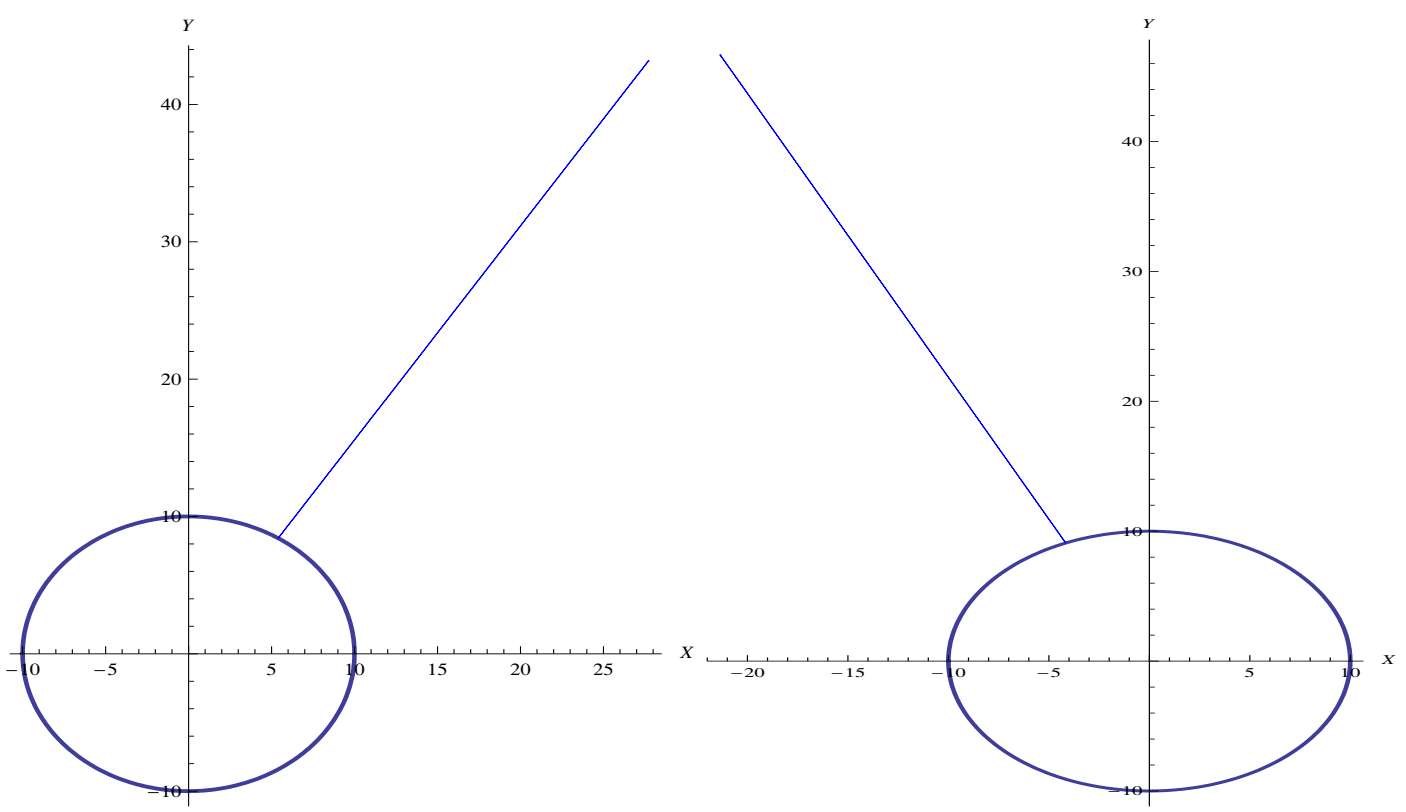

Figure 15. The position of string in $r-\phi$ plane at $\tau=1$ (left) and $\tau=2$ (right) plotted for $\sigma \in[-\pi, \pi], r_{+}=10$ (blue circle), $r_{-}=9$.

The space time coordinates for this embedding is given by

$$
r=\sqrt{\left(r_{+}^{2}-r_{-}^{2}\right)\left(\sinh ^{2} \sigma\right)+r_{+}^{2}}, \quad \phi=t=\frac{\tau}{r_{+}-r_{-}}
$$

where $r$ is again an even function of $\sigma$ and it's minimum occurs at $r_{+}$as seen in figure 14 . Figure 15 shows the snapshot of the string at two different $\tau$ 's. The open string is a spike which originates at the boundary, touches the horizon and returns back to the boundary. It is folded onto itself. Since $\phi$ is linear in $\tau$, the string will rotate around the horizon as $\tau$ changes. The end points of the string at the boundary describe a light like trajectory. 


\section{Conclusions}

We have applied the dressing method to construct and study classical string solutions in the BTZ background. Dressing time like and space like geodesics we obtained solutions which have dispersion relation similar to that of the giant magnon. Dressed time like geodesics are open strings whose end points move on time like trajectories with one point pinned to the boundary. They eventually fall into the horizon. We studied closed strings obtained by dressing space like geodesics. The minimal surfaces obtained are pinned on the boundary at two points and can cross the horizon. Giant gluons can be also embedded in the BTZ background. They give rise to two solutions, one of them is a spiral which expands and contracts, the other is a spike stretching from the boundary to the horizon.

There are several interesting directions which can be pursued to develop the observations of this paper further. It will be interesting to study the solutions obtained by multiple dressings and find their general properties, the appendix contains a discussion of the general procedure to obtain these solutions. If their dispersion relations turns out to be similar to multi-magnon solutions then perhaps these are the fundamental excitations of the integrable system corresponding to the BTZ background. Another direction is to investigate the role of these solutions from the boundary CFT point of view. The solution obtained by dressing space like geodesic is possibly best suited to be thought of as a nonlocal observable in the CFT. It will be interesting to pin down this observable precisely. Since these solutions penetrate the horizon while being pinned to the boundary, it will be extremely interesting to quantize the fluctuations about these solutions. This will help to verify the general predictions of the behaviour of strings near the horizon as argued in [22] and give more insight of the physics of extended objects near the horizon.

To summarize: classical string propagation in the BTZ sigma model is integrable. As shown in this paper it can be used to construct several interesting classical solutions in this background. This structure of the BTZ black hole is certainly worth further investigation.

\section{Acknowledgments}

We thank George Jorjadze, Chethan Krishnan, S. Prem Kumar, Gautam Mandal, Georgios Papathanasiou, Francisco Rojas and Aninda Sinha for useful comments and discussions. C.K thanks CHEP, IISc for hospitality during which this work was initiated. J.R.D and C.K thank the organizers of the "Recent Advances in Quantum Field Theory and String Theory" for a stimulating conference held at Tbilisi in 2011 during which some parts of this work was done. The work of J.R.D is partially supported by the Ramanujan fellowship DST-SR/S2/RJN-59/2009, the work of A.S is supported by a CSIR fellowship (File no: 09/079(2372)/2010-EMR-I).

\section{A $\quad N$-dressed geodesics}

Once we know a solution $\Psi_{0}(\lambda)$ to the system (2.20) it is possible to construct an explicit solution for the $N$-dressed geodesic. The details of this calculation for the $\mathrm{SU}(2)$ case have 
been worked out in [35]. A parallel treatment for $\mathrm{SU}(1,1)$ yields that the $N$-th dressed solution $g_{N}$ is

$$
g_{N}=\frac{\prod_{i=1}^{N} \frac{\lambda_{i}}{\left|\lambda_{i}\right|}}{\operatorname{det}\left(a_{i j}\right)}:\left|\begin{array}{cccc}
g_{0} & -h_{1} & \cdots & -h_{N} \\
h_{1}^{\dagger} M g_{0} & a_{11} & \cdots & a_{1 N} \\
\vdots & \vdots & \vdots & \vdots \\
h_{N}^{\dagger} M g_{0} & a_{N 1} & \cdots & a_{N N}
\end{array}\right|
$$

where the colons around the second determinant simply mean that upon expanding the determinant, the column $h_{i}$ is ordered before the row $h_{j}^{\dagger} M g_{0}$. In the above

$$
h_{i}=\Psi_{0}\left(\bar{\lambda}_{i}\right) e_{i}, \quad \alpha_{i j}=-\frac{\lambda_{i} \beta_{i j}}{\lambda_{i}-\bar{\lambda}_{j}}, \quad \beta_{i j}=h_{i}^{\dagger} M h_{j}
$$

The parameter $\lambda_{i}$ is the spectral parameter and $e_{i}$ the polarization vector.

As an example we give the first two dressed solutions

$$
\begin{aligned}
& g_{1}=\frac{\lambda_{1}}{\left|\lambda_{1}\right|}\left(1+\frac{h_{1} h_{1}^{\dagger} M}{\alpha_{11}}\right) g_{0}, \\
& g_{2}=\frac{\lambda_{1} \lambda_{2}}{\left|\lambda_{1} \lambda_{2}\right|}\left(1+\frac{\alpha_{22} h_{1} h_{1}^{\dagger} M+\alpha_{11} h_{2} h_{2}^{\dagger} M-\alpha_{12} h_{1} h_{2}^{\dagger} M-\alpha_{21} h_{2} h_{1}^{\dagger} M}{\alpha_{11} \alpha_{22}-\alpha_{12} \alpha_{21}}\right) g_{0} .
\end{aligned}
$$

In order to prove the above (A.1) we start by applying consecutive dressing transformations to the vacuum solution $\Psi_{0}(\lambda)$. Then, at the $N$-th step we will have $\Psi_{N}(\lambda)=$ $\chi_{N}(\lambda) \Psi_{N-1}(\lambda)$, with a dressing factor

$$
\chi_{N}(\lambda)=1+\frac{\lambda_{N}-\bar{\lambda}_{N}}{\lambda-\lambda_{N}} \mathcal{P}_{N}
$$

In the above, $\mathcal{P}_{N}$ is a rank one projection operator that can be expressed as

$$
\mathcal{P}_{N}=\frac{1}{\beta_{N N}^{(N-1)}} h_{N}^{(N-1)} h_{N}^{(N-1) \dagger} M
$$

where we have defined

$$
h_{i}^{(N)}=\Psi_{N}\left(\bar{\lambda}_{i}\right) e_{i}, \quad \beta_{i j}^{(N)}=h_{i}^{(N) \dagger} M h_{j}^{(N)}, \quad \alpha_{i j}^{(N)}=-\frac{\lambda_{i} \beta_{i j}^{(N)}}{\lambda_{i}-\bar{\lambda}_{j}},
$$

for any $N \geq 0$. We can then obtain a recursion relation for the matrix field $g_{N}=\Psi_{N}(0)$ which will be given by

$$
g_{N}=\frac{1}{\alpha_{N N}^{(N-1)}}\left(\alpha_{N N}^{(N-1)}+h_{N}^{(N-1)} h_{N}^{(N-1) \dagger} M\right) g_{N-1} .
$$


Furthermore, we can use the relations (A.4)-(A.7), and obtain the following recursion relations

$$
\begin{aligned}
h_{i}^{(N)} & =h_{i}^{(N-1)}-\frac{\alpha_{N i}^{(N-1)}}{\alpha_{N N}^{(N-1)}} h_{N}^{(N-1)} \\
\alpha_{i j}^{(N)} & =\alpha_{i j}^{(N-1)}-\frac{\alpha_{i N}^{(N-1)} \alpha_{N j}^{(N-1)}}{\alpha_{N N}^{(N-1)}} \\
h_{i}^{(N) \dagger} M g_{N} & =\frac{1}{\alpha_{N N}^{(N-1)}}\left(\alpha_{N N}^{(N-1)} h_{i}^{(N-1) \dagger} M g_{N-1}-\alpha_{i N}^{(N-1)} h_{N}^{(N-1) \dagger} M g_{N-1}\right),
\end{aligned}
$$

which after a straightforward calculation will lead to the final expression (A.1).

\section{References}

[1] S. Gubser, I. Klebanov and A.M. Polyakov, A Semiclassical limit of the gauge/string correspondence, Nucl. Phys. B 636 (2002) 99 [hep-th/0204051] [InSPIRE].

[2] A. Tseytlin, Review of AdS/CFT Integrability, Chapter II.1: Classical AdS $S_{5} \times S^{5}$ string solutions, Lett. Math. Phys. 99 (2012) 103 [arXiv:1012.3986] [INSPIRE].

[3] M. Spradlin and A. Volovich, Dressing the Giant Magnon, JHEP 10 (2006) 012 [hep-th/0607009] [INSPIRE].

[4] C. Kalousios, M. Spradlin and A. Volovich, Dressing the giant magnon II, JHEP 03 (2007) 020 [hep-th/0611033] [INSPIRE].

[5] A. Jevicki, C. Kalousios, M. Spradlin and A. Volovich, Dressing the Giant Gluon, JHEP 12 (2007) 047 [arXiv: 0708.0818] [INSPIRE].

[6] A. Jevicki, K. Jin, C. Kalousios and A. Volovich, Generating AdS String Solutions, JHEP 03 (2008) 032 [arXiv:0712.1193] [INSPIRE].

[7] J.R. David and B. Sahoo, Giant magnons in the D1 - D5 system, JHEP 07 (2008) 033 [arXiv: 0804.3267] [INSPIRE].

[8] A. Babichenko, B. Stefański Jr. and K. Zarembo, Integrability and the $A d S_{3} / C F T_{2}$ correspondence, JHEP 03 (2010) 058 [arXiv:0912.1723] [INSPIRE].

[9] J.R. David and B. Sahoo, S-matrix for magnons in the D1 - D5 system, JHEP 10 (2010) 112 [arXiv: 1005.0501] [INSPIRE].

[10] O. Ohlsson Sax and B. Stefański Jr., Integrability, spin-chains and the $A d S_{3} / C F T_{2}$ correspondence, JHEP 08 (2011) 029 [arXiv:1106.2558] [INSPIRE].

[11] P. Sundin and L. Wulff, Classical integrability and quantum aspects of the $A d S_{3} \times S^{3} \times S_{3} \times S_{1}$ superstring, JHEP 10 (2012) 109 [arXiv:1207.5531] [INSPIRE].

[12] O.O. Sax, B. Stefański Jr. and A. Torrielli, On the massless modes of the $A d S_{3} / C F T_{2}$ integrable systems, arXiv:1211.1952 [INSPIRE].

[13] C. Ahn and D. Bombardelli, Exact $S$-matrices for $A d S_{3} / C F T_{2}$, arXiv:1211.4512 [INSPIRE].

[14] R. Borsato, O.O. Sax and A. Sfondrini, A dynamic SU(1|1) ${ }^{2} S$-matrix for $A d S_{3} / C F T_{2}$, arXiv: 1211.5119 [INSPIRE].

[15] J.R. David and A. Sadhukhan, Classical integrability in the BTZ black hole, JHEP 08 (2011) 079 [arXiv: 1105. 0480] [INSPIRE]. 
[16] J. Louko, D. Marolf and S.F. Ross, On geodesic propagators and black hole holography, Phys. Rev. D 62 (2000) 044041 [hep-th/0002111] [INSPIRE].

[17] V. Balasubramanian and S.F. Ross, Holographic particle detection, Phys. Rev. D 61 (2000) 044007 [hep-th/9906226] [INSPIRE].

[18] P. Kraus, H. Ooguri and S. Shenker, Inside the horizon with AdS/CFT, Phys. Rev. D 67 (2003) 124022 [hep-th/0212277] [INSPIRE].

[19] L. Fidkowski, V. Hubeny, M. Kleban and S. Shenker, The Black hole singularity in AdS/CFT, JHEP 02 (2004) 014 [hep-th/0306170] [INSPIRE].

[20] G. Festuccia and H. Liu, Excursions beyond the horizon: Black hole singularities in Yang-Mills theories. I., JHEP 04 (2006) 044 [hep-th/0506202] [INSPIRE].

[21] T. Nishioka, S. Ryu and T. Takayanagi, Holographic Entanglement Entropy: An Overview, J. Phys. A 42 (2009) 504008 [arXiv: 0905.0932] [InSPIRE].

[22] L. Susskind, Strings, black holes and Lorentz contraction, Phys. Rev. D 49 (1994) 6606 [hep-th/9308139] [INSPIRE].

[23] A. Mezhlumian, A.W. Peet and L. Thorlacius, String thermalization at a black hole horizon, Phys. Rev. D 50 (1994) 2725 [hep-th/9402125] [INSPIRE].

[24] A.L. Larsen and A. Nicolaidis, String spreading on black hole horizon, Phys. Rev. D 60 (1999) 024012 [gr-qc/9812059] [INSPIRE].

[25] J.M. Maldacena, Wilson loops in large-N field theories, Phys. Rev. Lett. 80 (1998) 4859 [hep-th/9803002] [INSPIRE].

[26] S.-J. Rey, S. Theisen and J.-T. Yee, Wilson-Polyakov loop at finite temperature in large-N gauge theory and anti-de Sitter supergravity, Nucl. Phys. B 527 (1998) 171 [hep-th/9803135] [INSPIRE].

[27] A. Brandhuber, N. Itzhaki, J. Sonnenschein and S. Yankielowicz, Wilson loops in the large-N limit at finite temperature, Phys. Lett. B 434 (1998) 36 [hep-th/9803137] [INSPIRE].

[28] V.E. Hubeny, Extremal surfaces as bulk probes in AdS/CFT, JHEP 07 (2012) 093 [arXiv: 1203.1044] [INSPIRE].

[29] J.M. Maldacena and H. Ooguri, Strings in $A d S_{3}$ and $\mathrm{SL}(2, \mathbb{R})$ WZW model 1.: The Spectrum, J. Math. Phys. 42 (2001) 2929 [hep-th/0001053] [INSPIRE].

[30] D.M. Hofman and J.M. Maldacena, Giant Magnons, J. Phys. A 39 (2006) 13095 [hep-th/0604135] [INSPIRE].

[31] M. Kruczenski, A Note on twist two operators in $N=4$ SYM and Wilson loops in Minkowski signature, JHEP 12 (2002) 024 [hep-th/0210115] [INSPIRE].

[32] L.F. Alday and J.M. Maldacena, Gluon scattering amplitudes at strong coupling, JHEP 06 (2007) 064 [arXiv: 0705. 0303] [INSPIRE].

[33] M. Bañados, C. Teitelboim and J. Zanelli, The Black hole in three-dimensional space-time, Phys. Rev. Lett. 69 (1992) 1849 [hep-th/9204099] [INSPIRE].

[34] M. Bañados, M. Henneaux, C. Teitelboim and J. Zanelli, Geometry of the $(2+1)$ black hole, Phys. Rev. D 48 (1993) 1506 [gr-qc/9302012] [InSPIRE].

[35] C. Kalousios and G. Papathanasiou, Giant Magnons in Symmetric Spaces: Explicit N-soliton solutions for $C P^{n}, \mathrm{SU}(N)$ and $S^{n}$, JHEP 07 (2010) 068 [arXiv:1005.1066] [INSPIRE]. 